TRANSACTIONS OF THE

AMERICAN MATHEMATICAL SOCIETY

Volume 360, Number 8, August 2008, Pages 4287-4304

S 0002-9947(08)04475-9

Article electronically published on March 12, 2008

\title{
CONTINUED FRACTIONS WITH CIRCULAR TWIN VALUE SETS
}

\author{
LISA LORENTZEN
}

\begin{abstract}
We prove that if the continued fraction $K\left(a_{n} / 1\right)$ has circular twin value sets $\left\langle V_{0}, V_{1}\right\rangle$, then $K\left(a_{n} / 1\right)$ converges except in some very special cases. The results generalize previous work by Jones and Thron.
\end{abstract}

\section{INTRODUCTION AND MAIN RESULT}

A pair $\left\langle V_{0}, V_{1}\right\rangle$ of sets from $\widehat{\mathbb{C}}:=\mathbb{C} \cup\{\infty\}$ is called a pair of twin value sets for the continued fraction

$$
K\left(a_{n} / 1\right):=\frac{a_{1}}{1}+\frac{a_{2}}{1}+\frac{a_{3}}{1}+\ldots:=\frac{a_{1}}{1+\frac{a_{2}}{1+\frac{a_{3}}{1+\ldots}}}, \quad a_{n} \in \mathbb{C} \backslash\{0\}
$$

if both $V_{k}$ and its complement $V_{k}^{c}$ in $\widehat{\mathbb{C}}$ are non-empty for $k=0,1$ and

$$
a_{2 n-1} /\left(1+V_{1}\right) \subseteq V_{0} \quad \text { and } \quad a_{2 n} /\left(1+V_{0}\right) \subseteq V_{1} \quad \text { for } n=1,2,3, \ldots
$$

Note that we do not require $a_{2 n+k} \in V_{k-1}$ for $k=1,2$ as was done in the work by Jones and Thron; see for instance their book [7, p. 64]. For given value sets we further define the corresponding element sets $\left\langle E_{1}, E_{2}\right\rangle$ by

$$
E_{1}:=\left\{a \in \mathbb{C} ; a /\left(1+V_{1}\right) \subseteq V_{0}\right\}, \quad E_{2}:=\left\{a \in \mathbb{C} ; a /\left(1+V_{0}\right) \subseteq V_{1}\right\} .
$$

Here, by definition, $0 \notin E_{1}$ if $-1 \in \overline{V_{1}}$ (the closure of $V_{1}$ in $\widehat{\mathbb{C}}$ ) and $0 \notin E_{2}$ if $-1 \in \overline{V_{0}}$. The twin element sets $\left\langle E_{1}, E_{2}\right\rangle$ are true if $E_{k} \backslash\{0\} \neq \emptyset$ for $k=1$ and 2 . We also say that $\left\langle V_{0}, V_{1}\right\rangle$ are twin value sets for $\left\langle E_{1}, E_{2}\right\rangle$. For convenience we shall always let $V_{2}:=V_{0}$, so that $E_{k}=\left\{a \in \mathbb{C} ; a /\left(1+V_{k}\right) \subseteq V_{k-1}\right\}$ for $k=1,2$.

In this paper we restrict the value sets to be closed circular domains; that is, they are closures of simply connected, open, non-empty domains on the Riemann sphere $\widehat{\mathbb{C}}$, bounded by a generalized circle. The points $0,-1, \infty$ are special in the classical continued fraction theory. (See (1.6).) We shall therefore distinguish between closed domains $V$ where

- $\infty \notin V$ (disks),

- $\infty$ on the boundary $\partial V$ of $V$ (half planes),

- $\infty$ in the interior $V^{\circ}$ of $V$ (complements of disks).

Received by the editors December 4, 2005 and, in revised form, August 16, 2006. 2000 Mathematics Subject Classification. Primary 40A15.

(C)2008 American Mathematical Society 
We address the problem: when does $K\left(a_{n} / 1\right)$ from $\left\langle E_{1}, E_{2}\right\rangle$ (i.e. all $a_{2 n-1} \in E_{1}$ and all $\left.a_{2 n} \in E_{2}\right)$ converge? By convergence we mean that the sequence of approximants $\left\{c_{n}\right\}$ of $K\left(a_{n} / 1\right)$ converges to a $c \in \widehat{\mathbb{C}}$, where

$$
\begin{aligned}
& c_{n}:=S_{n}(0) \quad \text { and } \quad S_{n}(z):=\frac{a_{1}}{1}+\frac{a_{2}}{1}+\cdots+\frac{a_{n}}{1+z}, \\
& \text { i.e., } \quad S_{n}:=s_{0} \circ s_{1} \circ s_{2} \circ \cdots \circ s_{n} ; \quad s_{0}(z):=z, s_{k}(z):=\frac{a_{k}}{1+z} .
\end{aligned}
$$

We say that the even (odd) part of $K\left(a_{n} / 1\right)$ converges if $\left\{c_{2 n}\right\}\left(\left\{c_{2 n+1}\right\}\right)$ converges in $\widehat{\mathbb{C}}$. A number of papers has been written on this topic. See for instance [7, chapter 4] and the references therein. In particular, the paper [6 by Jones and Thron, published in this journal, gives a very nice and useful presentation of sufficient conditions for convergence. However, these results can be improved, as we shall show in this paper. The very special case where $0 \in \partial V_{0}$ and $-1 \in \partial V_{1}$ or vice versa still needs some extra attention, though (see Example 2.9). We shall prove:

Theorem 1.1. Let $\left\langle V_{0}, V_{1}\right\rangle$ be closed circular twin value sets with corresponding element sets $\left\langle E_{1}, E_{2}\right\rangle$ for the continued fraction $K\left(a_{n} / 1\right)$. Then the following statements are true:

A. Let $V_{0}$ and $V_{1}$ be disks and $E_{2}^{\circ} \neq \emptyset$. Then $K\left(a_{n} / 1\right)$ converges to a $c \in V_{0}$.

B. Let $V_{0}$ be a disk, $V_{1}$ be a half plane and $E_{2}^{\circ} \neq \emptyset$. Then $K\left(a_{n} / 1\right)$ converges to $a c \in V_{0}$.

C. Let $V_{0}$ be a disk and $V_{1}$ be the complement of a disk with respective centers $C_{k}$ and radii $R_{k}$ such that $\left|C_{k}\right| R_{k+1} \neq R_{k}\left|1+C_{k+1}\right|$ for $k=0$ or $k=1$ and $0 \notin \partial^{\dagger} V_{1}:=\partial V_{1} \cap\left(-1-\partial V_{0}\right)$. Then the even part of $K\left(a_{n} / 1\right)$ converges to $a c \in V_{0}$. If moreover $-1 \notin V_{0} \backslash\left(-1-V_{1}^{\circ}\right)$, then $K\left(a_{n} / 1\right)$ itself converges to $c$.

D. Let $V_{0}$ and $V_{1}$ be half planes with $0,-1 \notin \partial^{\dagger} V_{1}$. Then the even and odd parts of $K\left(a_{n} / 1\right)$ converge to finite values $\in V_{0}$. Moreover, $K\left(a_{n} / 1\right)$ itself converges if and only if

$$
\sum_{n=1}^{\infty}\left|b_{n}\right|=\infty \quad \text { where } b_{2 n}:=\frac{a_{1} a_{3} \cdots a_{2 n-1}}{a_{2} a_{4} \cdots a_{2 n}}, \quad b_{2 n+1}:=\frac{a_{2} a_{4} \cdots a_{2 n}}{a_{1} a_{3} \cdots a_{2 n+1}} .
$$

Remarks 1.2.

1. Since $K_{n=1}^{\infty}\left(a_{n} / 1\right)$ converges in $\widehat{\mathbb{C}}$ if and only if $K_{n=2}^{\infty}\left(a_{n} / 1\right)$ converges in $\widehat{\mathbb{C}}$, we may interchange $V_{0}$ and $V_{1}$.

2. Theorem 1.1 also covers cases such as, for instance, $V_{0}$ a half plane and $V_{1}$ a complement of a disk, since $\left\langle V_{0}, V_{1}\right\rangle$ are twin value sets for the continued fraction $K\left(a_{n} / 1\right)$ if and only if $\left\langle-1-V_{1}^{c},-1-V_{0}^{c}\right\rangle$ are twin value sets for $K\left(a_{n} / 1\right)$ (see Lemma 4.1). This was also pointed out by Jones and Thron in 6]. Indeed, if $V_{0}$ or $V_{1}$ contains more than one element, then $Y_{0}:=V_{0} \backslash\left(-1-V_{1}\right)^{\circ} \neq \emptyset$ and $Y_{1}:=V_{1} \backslash\left(-1-V_{0}\right)^{\circ} \neq \emptyset$, so also $\left\langle Y_{0}, Y_{1}\right\rangle$ are twin value sets for $K\left(a_{n} / 1\right)$, [9, prop. 5.4].

3. It is a well established fact [7, thm. 4.53, p. 128] that (1.5) holds if $\left\{a_{n}\right\}$ has a bounded subsequence.

The classical convergence concept requires that $S_{n}(0) \rightarrow c$, where by (1.4),

$$
c_{n}=S_{n-1}\left(a_{n}\right)=S_{n}(0)=S_{n+1}(\infty)=S_{n+2}(-1)=S_{n+3}\left(-1-a_{n+3}\right) .
$$


In 2] a more general concept of convergence was introduced: we require that there exist two sequences $\left\{u_{n}\right\}$ and $\left\{v_{n}\right\}$ from $\widehat{\mathbb{C}}$ such that

$$
\lim S_{n}\left(u_{n}\right)=\lim S_{n}\left(v_{n}\right)=c \quad \text { and } \liminf d\left(u_{n}, v_{n}\right)>0,
$$

where $d(*, *)$ denotes the chordal metric on the Riemann sphere $\widehat{\mathbb{C}}$; i.e.,

$$
d(z, w)=\frac{2|z-w|}{\sqrt{1+|z|^{2}} \sqrt{1+|w|^{2}}} \quad \text { if } z, w \in \mathbb{C}
$$

with the natural limit forms if $z$ and/or $w$ is $=\infty$. If (1.7) holds, we say that $K\left(a_{n} / 1\right)$ converges generally to $c$. Then, by [2, there exists an exceptional sequence $\left\{z_{n}^{\dagger}\right\} \subseteq \widehat{\mathbb{C}}$ such that

$$
\lim S_{n}\left(z_{n}\right)=c \quad \text { whenever } \liminf d\left(z_{n}, z_{n}^{\dagger}\right)>0 .
$$

If $c \neq \infty$, we can for instance use $z_{n}^{\dagger}:=\zeta_{n}:=S_{n}^{-1}(\infty)$ for all $n$. Or more generally, $\left\{S_{n}^{-1}(q)\right\}$ is an exceptional sequence for every $q \neq c$, also if $c=\infty$. All the exceptional sequences have the same asymptotic behavior.

Classical convergence implies general convergence whereas the converse does not hold. Indeed, there are generally convergent continued fractions $K\left(a_{n} / 1\right)$ where $\left\{z_{n}^{\dagger}\right\}$ has limit points at $0,-1$ and $\infty$ which destroy the classical convergence of $K\left(a_{n} / 1\right)$. However, if $K\left(a_{n} / 1\right)$ also converges in the classical sense, then it converges to the same value. It is also clear that if the even and odd parts of $K\left(a_{n} / 1\right)$ converge to distinct values in the classical sense, then they also converge generally to the same two distinct values.

One might expect to get a nicer theorem with general convergence. However, Theorem 1.1 is already good, except for the disk - complement of disk case. For this case it really pays off to change over to general convergence (here $B(C, R)$ denotes a closed circular disk with center at $C \in \mathbb{C}$ and radius $R>0$ ):

Theorem 1.3. Let $V_{0}:=B\left(C_{0}, R_{0}\right)$ and $V_{1}:=\overline{B\left(C_{1}, R_{1}\right)^{c}}$ be twin value sets for the continued fraction $K\left(a_{n} / 1\right)$, where $0 \notin \partial^{\dagger} V_{1}:=\partial V_{1} \cap\left(-1-\partial V_{0}\right)$ and $\left|C_{k}\right| R_{k+1} \neq R_{k}\left|1+C_{k+1}\right|$ for $k=0$ or $k=1$. Then $K\left(a_{n} / 1\right)$ converges generally to a $c \in V_{0}$.

The final result in this section describes cases where classical convergence follows from general convergence. We still use the notation $\zeta_{n}:=S_{n}^{-1}(\infty)$.

Theorem 1.4. Let $\left\langle V_{0}, V_{1}\right\rangle$ be closed twin value sets for the continued fraction $K\left(a_{n} / 1\right)$ with $\left(V_{0} \cup V_{1}\right)^{\circ} \neq \emptyset$. Let $K\left(a_{n} / 1\right)$ converge generally to $c$, let $q \neq c$ and let $\tilde{Z}_{k}$ be the set of limit points for $\left\{S_{2 n+k}^{-1}(q)\right\}$. Then the following statements hold for fixed $k \in\{1,2\}$.

A. $c \in V_{0} \backslash\left(-1-V_{1}^{\circ}\right)$ and $\tilde{Z}_{k} \subseteq\left(-1-V_{k-1}\right) \backslash V_{k}^{\circ}$.

B. If $-1 \notin \tilde{Z}_{k}$ or $0 \notin \tilde{Z}_{k}$, then $S_{2 n+k}(0) \rightarrow c$. If $\infty \notin \tilde{Z}_{k}$, then $S_{2 n+k-1}(0) \rightarrow$ c.

C. Let $\varepsilon>0$ and $n_{0} \in \mathbb{N}$. If for each $n \geq n_{0}$, either $d\left(a_{2 n+k-1}, \tilde{Z}_{k}\right) \geq \varepsilon$ or $d\left(-1-a_{2 n+k+2}, \tilde{Z}_{k}\right) \geq \varepsilon$, then $S_{2 n+k-1}(0) \rightarrow c$.

D. If $V_{0}$ is bounded, then $\left\{\zeta_{n}\right\}$ is an exceptional sequence for $\left\{S_{n}\right\}$ and $S_{2 n}(0)$ $\rightarrow c$.

E. If $-1 \notin V_{0} \backslash\left(-1-V_{1}^{\circ}\right)$, then $S_{2 n+1}(0) \rightarrow c$. 
In section 2 we shall give some explicit expressions for the corresponding element sets $\left\langle E_{1}, E_{2}\right\rangle$ and some stronger convergence results. Section 3 contains some intermediate results, and in section 4 we prove the results in sections 1 and 2 .

Notation. We shall use the notation introduced so far, plus some extra. For convenience we list a few of them here:

- $\bar{A}, A^{\circ}, \partial A$ and $A^{c}$ are the closure, the interior, the boundary and the complement of a set $A$ in $\widehat{\mathbb{C}}$.

- $\mathbb{D}$ is the open unit disk $\{z \in \mathbb{C} ;|z|<1\}$.

- $\left[z_{1}, z_{2}\right]$ is the closed line segment between the two points $z_{1}$ and $z_{2}$ in $\mathbb{C}$. Moreover, $a[r, \infty):=\{z=u a ; u \geq r\}$ for $a \in \mathbb{C} \backslash\{0\}$ and $r \in \mathbb{R}$.

- $B(a, r):=\{z \in \mathbb{C} ;|z-a| \leq r\}$ and $B_{d}(a, r):=\{z \in \widehat{\mathbb{C}} ; d(z, a) \leq r\}$ for $a \in \mathbb{C}$ and $r>0$.

- $H(r, \alpha)$, where $r, \alpha \in \mathbb{R}$, denotes the closed half plane with $L:=e^{i \alpha}[r, \infty) \subseteq$ $H(r, \alpha)$, whose boundary $\partial H(r, \alpha)$ is the line through $r e^{i \alpha}$ orthogonal to $L$.

- $\operatorname{rad}(A)$ is the euclidean radius of a circular set $A \subseteq \widehat{\mathbb{C}} \cdot \operatorname{rad}(A):=\infty$ if $\infty \in \bar{A}$.

- $\operatorname{diam}(A)$ is the euclidean diameter of a set $A \subset \widehat{\mathbb{C}}$.

- $\operatorname{dist}(z, A)(d(z, A))$ denotes the euclidean (chordal) distance between a point $z \in \widehat{\mathbb{C}}$ and a set $A \subseteq \widehat{\mathbb{C}}$, and $\operatorname{dist}(A, B)(d(A, B))$ denotes the euclidean (chordal) distance between two sets $A, B \subseteq \widehat{\mathbb{C}}$.

- For convenience, $V_{2}:=V_{0}, W_{2}:=W_{0}, E_{3}:=E_{1}, E_{0}=E_{2}$, etc. for twin quantities; that is, they are counted modulo 2.

- $s_{m}$ denotes the linear fractional transformation $a_{m} /(1+z), s_{m}^{*}(z):=a_{m}^{*} /$ $(1+z)$ and so on, and $S_{n}:=s_{1} \circ s_{2} \circ \cdots \circ s_{n}$.

- $\partial^{\dagger} V_{k}:=\partial V_{k} \cap\left(-1-\partial V_{k+1}\right)$ and $\partial^{*} V_{k}:=\partial V_{k} \cap\left(-1-V_{k+1}\right)$ for $k=0,1$. Clearly, $\partial^{\dagger} V_{0}=-1-\partial^{\dagger} V_{1}$, and the condition $0 \notin \partial V_{k},-1 \notin \partial V_{k+1}$ can be written $0 \notin \partial^{\dagger} V_{k}$, or equivalently, $-1 \notin \partial^{\dagger} V_{k+1}$.

- $\zeta_{n}:=S_{n}^{-1}(\infty), c_{n}:=S_{n}(0)$ and $Z_{k}$ is the (closed) set of limit points for $\left\{\zeta_{2 n+k}\right\}$.

- $W_{0}:=-1-\overline{V_{1}^{c}}, W_{1}:=-1-\overline{V_{0}^{c}}, Y_{0}:=V_{0} \backslash\left(-1-V_{1}\right)^{\circ}$ and $Y_{1}:=V_{1} \backslash$ $\left(-1-V_{0}\right)^{\circ}$ so that $\left\langle W_{0}, W_{1}\right\rangle$ and $\left\langle Y_{0}, Y_{1}\right\rangle$ are alternative closed twin value sets (Remark 1.2.2).

- $\sum^{\prime} P_{n}<\infty$ shall mean that there exists an $n_{0} \in \mathbb{N}$ such that $\sum_{n=n_{0}}^{\infty} P_{n}<$ $\infty$ for the non-negative numbers $P_{n}$. Hence $P_{n}=\infty$ is possible for finitely many $n$.

\section{EXPLiCIT ELEMENT SETS AND MORE DETAILED CONVERGENCE CRITERIA}

In applications it is useful to know the corresponding element sets $\left\langle E_{1}, E_{2}\right\rangle$ explicitly. We have therefore listed these sets below, along with some more specific convergence criteria for continued fractions $K\left(a_{n} / 1\right)$ with circular twin value sets. Of course we want as few extra conditions as possible, but some situations have to be treated separately:

- $a_{n} \rightarrow \infty$. The if and only if part of Theorem 1.1D shows that extra conditions are needed in this case. This is true whether we want classical or general convergence. 
- $a_{2 n-1} \rightarrow \tilde{a}_{1} \in E_{1} \backslash\{0\}$ and $a_{2 n} \rightarrow \tilde{a}_{2} \in E_{2} \backslash\{0\}$ where $\tilde{s}_{1} \circ \tilde{s}_{2}$ is an elliptic transformation. If $\left|a_{2 n+k}-\tilde{a}_{k}\right| \rightarrow 0$ fast enough for $k=1$ and $k=2$, then $K\left(a_{n} / 1\right)$ diverges generally. $\tilde{s}_{1} \circ \tilde{s}_{2}$ is elliptic if $\tilde{a}_{1}=-w_{0} w_{1}$ and $\tilde{a}_{2}=-\left(1+w_{0}\right)\left(1+w_{1}\right)$ for some $w_{0}, w_{1} \in \mathbb{C}$ with $w_{0}\left(1+w_{1}\right)=$ $e^{i \theta} w_{1}\left(1+w_{0}\right)$ where $e^{i \theta} \neq 1$, [1]. This can happen only if both $\tilde{s}_{1}\left(V_{1}\right)=V_{0}$ and $\tilde{s}_{2}\left(V_{0}\right)=V_{1}$. (See also Lemma 4.2.)

- $\tilde{a}_{k}:=0 \in \overline{E_{k}}$ and $\tilde{a}_{k+1}:=-1 \in E_{k+1}$ for $k=1$ or 2 . Also now $K\left(a_{n} / 1\right)$ with $a_{2 n+k} \rightarrow \tilde{a}_{k}$ for $k=1,2$ may converge or diverge depending on how $\left\{a_{2 n+k}\right\}$ approaches $\tilde{a}_{k}$ (see Example 2.9).

The disk - disk case.

Let $V_{k}:=B\left(C_{k}, R_{k}\right)$ for some $C_{k} \in \mathbb{C}$ and $R_{k}>0$ for $k=0,1$. Evidently $E_{k}=\emptyset$ if $-1 \in V_{k}$, so

$$
\left|1+C_{k}\right|>R_{k} \quad \text { for } k=0,1
$$

is a necessary condition for $\left\langle E_{1}, E_{2}\right\rangle$ to be true element sets corresponding to $\left\langle V_{0}, V_{1}\right\rangle$. Then we get the following generalization of [6, thm. 5.1]:

Theorem 2.1. Let $V_{k}:=B\left(C_{k}, R_{k}\right)$ for $k=0,1$ where $C_{k} \in \mathbb{C}$ and $R_{k}>0$ satisfy (2.1) and

$$
\left|C_{k-1}\right| R_{k} \leq\left|1+C_{k}\right| R_{k-1}
$$

for $k=1,2$. If (2.2) holds with equality for both $k=1$ and $k=2$, we further assume that $\sigma:=\tilde{s}_{1} \circ \tilde{s}_{2}$ is non-elliptic, where

$$
\tilde{a}_{k}:=C_{k-1}\left(1+C_{k}\right)\left(1-R_{k}^{2} /\left|1+C_{k}\right|^{2}\right) .
$$

Then every continued fraction $K\left(a_{n} / 1\right)$ from $\left\langle E_{1}, E_{2}\right\rangle$ converges, where

$$
E_{k}:=\left\{a \in \mathbb{C} ;\left|a-\tilde{a}_{k}\right|+\frac{R_{k}}{\left|1+C_{k}\right|}|a| \leq \frac{R_{k-1}}{\left|1+C_{k}\right|}\left(\left|1+C_{k}\right|^{2}-R_{k}^{2}\right)\right\} .
$$

Remarks 2.2.

1. $\left\langle E_{1}, E_{2}\right\rangle$ are the element sets corresponding to $\left\langle V_{0}, V_{1}\right\rangle$. They are true element sets if and only if (2.1) and (2.2) hold. Condition (2.2) is therefore only present to make $\left\langle E_{1}, E_{2}\right\rangle$ true when (2.1) holds. $E_{k}$ is a one-point set if and only if $E_{k}=\left\{\tilde{a}_{k}\right\}$ as given by (2.3). This happens if and only if $\left|C_{k-1}\right| R_{k}=\left|1+C_{k}\right| R_{k-1}$, which happens if and only if $a /\left(1+V_{k}\right)=V_{k-1}$ for an $a \in E_{k}$, in which case $a=\tilde{a}_{k} \neq 0$. (See Lemma 4.3.)

2. If $E_{k}$ contains more than one point, then $E_{k}$ is a closed convex domain bounded by a cartesian oval with foci at 0 and $\tilde{a}_{k}$ [3, 12, remark 5, p. 142], and $E_{k}^{\circ} \neq \emptyset$. If $C_{k-1}=0$, this oval reduces to a circle centered at the origin.

3. Divergence only occurs if and only if $E_{k}=\left\{\tilde{a}_{k}\right\}$ for $k=1,2$ and $\sigma:=\tilde{s}_{1} \circ \tilde{s}_{2}$ is elliptic. This means that $K\left(a_{n} / 1\right)$ converges in the classical sense if and only if it converges in the general sense in the disk-disk case.

The disk - half plane case.

Let $V_{0}:=B\left(C_{0}, R_{0}\right)$ and $V_{1}:=\left\{z \in \mathbb{C} ; \operatorname{Re}\left(z e^{-i \alpha}\right) \geq h \cos \alpha\right\} \cup\{\infty\}=$ $H(h \cos \alpha, \alpha)$ for some $C_{0} \in \mathbb{C}, R_{0}>0, h, \alpha \in \mathbb{R}$. It is clear that $a /\left(1+V_{1}\right) \subseteq V_{0}$ for $a \neq 0$ only if $-1 \notin V_{1}$ and $0 \in V_{0}$, and that $a /\left(1+V_{0}\right) \subseteq V_{1}$ for $a \neq 0$ only if $-1 \notin V_{0}^{\circ}$. Hence we require that

$$
\left|C_{0}\right| \leq R_{0} \leq\left|1+C_{0}\right|, \quad|\alpha|<\pi / 2 \quad \text { and } \quad h>-1 .
$$


But this leaves the possibility of $0 \in \partial V_{1}$ and $-1 \in \partial V_{0}$, a situation that requires caution. We therefore need extra conditions if $0 \in \partial^{\dagger} V_{1}:=\partial V_{1} \cap\left(-1-\partial V_{0}\right)$. Still, we get the following generalization of [6, thm. 5.2]:

Theorem 2.3. Let $V_{0}:=B\left(C_{0}, R_{0}\right)$ and $V_{1}:=H(h \cos \alpha, \alpha)$ where $C_{0} \in \mathbb{C}, R_{0}>0$ and $\alpha, h \in \mathbb{R}$ satisfy (2.5), and let

$$
a_{1}^{*}:=2 C_{0} e^{i \alpha}(1+h) \cos \alpha, \quad a_{2}^{*}:=2\left(1+C_{0}\right) h e^{i \alpha} \cos \alpha
$$

and

$$
\begin{aligned}
E_{1} & :=\left\{a \in \mathbb{C} ;\left|a-a_{1}^{*}\right|+|a| \leq 2 R_{0}(1+h) \cos \alpha\right\}, \\
(2.7)_{E_{2}} & := \begin{cases}\left\{a \in \mathbb{C} ;|a| R_{0}-\operatorname{Re}\left(a\left(1+\overline{C_{0}}\right) e^{-i \alpha}\right) \leq-h\left(\left|1+C_{0}\right|^{2}-R_{0}^{2}\right) \cos \alpha\right\} \\
\left(1+C_{0}\right) e^{i \alpha}[\max \{0,2 h \cos \alpha\}, \infty) \backslash\{0\} & \text { if }\left|1+C_{0}\right|>R_{0},\end{cases}
\end{aligned}
$$

Furthermore, let

$$
\begin{aligned}
& \tilde{E}_{1, \delta}:= \begin{cases}E_{1} \backslash B\left(a_{1}^{*}, \delta\right) & \text { if } R_{0}=\left|C_{0}\right|, \\
E_{1} & \text { otherwise, }\end{cases} \\
& \tilde{E}_{2, \delta}:= \begin{cases}E_{2} \backslash B\left(a_{2}^{*}, \delta\right) & \text { if } R_{0}=\left|1+C_{0}\right| \text { and } h \geq 0, \\
E_{2} & \text { otherwise }\end{cases}
\end{aligned}
$$

where $0<\delta<\left|a_{1}^{*}\right|$ if $C_{0} \neq 0$. Then the following statements are true:

A. Every continued fraction $K\left(a_{n} / 1\right)$ from $\left\langle E_{1}, \tilde{E}_{2, \delta}\right\rangle$ converges generally.

B. If $0 \notin \partial^{\dagger} V_{1}$, then every continued fraction $K\left(a_{n} / 1\right)$ from $\left\langle\tilde{E}_{1, \delta}, E_{2}\right\rangle$ converges generally.

C. Let $\varepsilon>0$. If $K\left(a_{n} / 1\right)$ is a continued fraction from $\left\langle\tilde{E}_{1, \delta}, E_{2}\right\rangle$ such that for each $n$ from some $n_{0}$ on, either $\operatorname{dist}\left(-1-a_{2 n}, \partial^{*} V_{0}\right) \geq \varepsilon$ or $\operatorname{dist}\left(a_{2 n-1}, \partial^{*} V_{0}\right)$ $\geq \varepsilon$, then $K\left(a_{n} / 1\right)$ converges generally.

D. Let $K\left(a_{n} / 1\right)$ from $\left\langle E_{1}, E_{2}\right\rangle$ converge generally to $c$. Then $c_{2 n} \rightarrow c$. If moreover $0 \in V_{1}^{\circ}$ or $-1 \notin \partial V_{0}$ or $\liminf d\left(a_{2 n-1}, \overline{V_{0}^{c}} \cap\left(-1-V_{1}\right)\right)>0$, then $c_{n} \rightarrow c$.

Remarks 2.4 .

1. $\left\langle E_{1}, E_{2}\right\rangle$ are the element sets corresponding to $\left\langle V_{0}, V_{1}\right\rangle$. If $R_{0}=\left|C_{0}\right|$, then $E_{1}$ is the closed line segment $\left[0, a_{1}^{*}\right]$. Otherwise, $\partial E_{1}$ is an ellipse with foci at $a_{1}^{*}$ and the origin. $\partial E_{1}$ reduces to a circle if $C_{0}=0$.

2. If $\left|1+C_{0}\right|=R_{0}$, then $E_{2}$ is a ray. Otherwise, $E_{2}^{\circ} \neq \emptyset$ and $\partial E_{2}$ is a hyperbola.

3. If $-1 \notin \partial V_{0}$ or $0 \in V_{1}^{\circ}$, then $\tilde{E}_{2, \delta}=E_{2}$, so every continued fraction $K\left(a_{n} / 1\right)$ from $\left\langle E_{1}, E_{2}\right\rangle$ converges generally by part $\mathrm{A}$ in this case. Let $-1 \in \partial V_{0}$ and $0 \notin V_{1}^{\circ}$. If $0 \notin \partial V_{1}$ and $0 \in V_{0}^{\circ}$, then $\tilde{E}_{1, \delta}=E_{1}$, and every continued fraction from $\left\langle E_{1}, E_{2}\right\rangle$ still converges generally by part B.

The disk - complement of disk case.

Let $V_{0}=B\left(C_{0}, R_{0}\right)$ and $V_{1}=\overline{B\left(C_{1}, R_{1}\right)^{c}}$. This time $\infty \notin V_{0}$ and $\infty \in V_{1}^{\circ}$, so we evidently need that $0 \in V_{0}^{\circ}$ and $-1 \notin V_{1}$ to get true element sets; that is,

$$
\left|C_{0}\right|<R_{0} \quad \text { and } \quad\left|1+C_{1}\right|<R_{1} \text {. }
$$


Theorem 2.5. Let $V_{0}:=B\left(C_{0}, R_{0}\right)$ and $V_{1}:=\overline{B\left(C_{1}, R_{1}\right)^{c}}$ where $C_{k} \in \mathbb{C}$ and $R_{k}>0$ satisfy (2.9), and let

$$
\begin{aligned}
& E_{1}:=\left\{\begin{array}{lr}
\left\{a ;\left|a-\tilde{a}_{1}\right|+|a| \frac{R_{1}}{\left|1+C_{1}\right|} \leq \frac{R_{0}}{\left|1+C_{1}\right|}\left(R_{1}^{2}-\left|1+C_{1}\right|^{2}\right)\right\} & \text { if } C_{1} \neq-1, \\
B\left(0,\left(R_{0}-\left|C_{0}\right|\right) R_{1}\right) & \text { if } C_{1}=-1,
\end{array}\right. \\
& E_{2}:=\left\{\begin{array}{lr}
\left\{a ;\left|a-\tilde{a}_{2}\right|-|a| \frac{R_{0}}{\left|1+C_{0}\right|} \geq \frac{R_{1}}{\left|1+C_{0}\right|}\left(\left|1+C_{0}\right|^{2}-R_{0}^{2}\right)\right\} \backslash\{0\} & \text { if } R_{0}<\left|1+C_{0}\right|, \\
\left\{a ;|a| \frac{R_{0}}{\left|1+C_{0}\right|}-\left|a-\tilde{a}_{2}\right| \geq \frac{R_{1}}{\left|1+C_{0}\right|}\left(R_{0}^{2}-\left|1+C_{0}\right|^{2}\right)\right\} & \text { if } R_{0}>\left|1+C_{0}\right|>0, \\
\left\{a=r e^{i \theta} ; \frac{r}{2} \geq \operatorname{Re}\left(C_{1}\left(1+C_{0}\right) e^{-i \theta}\right)+R_{0} R_{1}\right\} \backslash\{0\} & \text { if } R_{0}=\left|1+C_{0}\right|, \\
\left\{a ;|a| \geq R_{0}\left(R_{1}+\left|C_{1}\right|\right)\right\} & \text { if } C_{0}=-1,
\end{array}\right.
\end{aligned}
$$

where $\tilde{a}_{k}$ is given by (2.3). Further let $\widehat{E}_{1, \delta}$ be given by (2.11), and let $\widehat{E}_{2, \delta}:=E_{2}$ if $-1 \notin V_{0}^{\circ}$ and $\widehat{E}_{2, \delta}$ be given by (2.11) otherwise, where

$$
\widehat{E}_{k, \delta}:=\left\{\begin{array}{lc}
E_{k} \backslash B\left(\tilde{a}_{k}, \delta\right)^{\circ} & \text { if }\left|C_{k-1}\right| R_{k}=R_{k-1}\left|1+C_{k}\right|>0, \\
E_{k} \backslash\left\{a \in \mathbb{C} ;|| a\left|-R_{0} R_{1}\right|<\delta\right\} & \text { if } C_{k-1}=1+C_{k}=0, \\
E_{k} & \text { otherwise }
\end{array}\right.
$$

for given $\delta>0$ so small that $\widehat{E}_{1, \delta} \neq \emptyset$. Then the following statements are true.

A. $\left\langle E_{1}, E_{2}\right\rangle$ are the element sets corresponding to $\left\langle V_{0}, V_{1}\right\rangle$, and $E_{k}^{\circ} \neq \emptyset$ for $k=1,2$.

B. Let $0 \notin \partial^{\dagger} V_{1}$. Then every continued fraction $K\left(a_{n} / 1\right)$ from $\left\langle\widehat{E}_{1 \delta}, E_{2}\right\rangle$ or from $\left\langle E_{1}, \widehat{E}_{2, \delta}\right\rangle$ converges generally.

C. Let $\varepsilon>0$. If $K\left(a_{n} / 1\right)$ is a continued fraction from $\left\langle\widehat{E}_{1, \delta}, E_{2},\right\rangle$ such that for each $n$ from some $n_{0} \in \mathbb{N}$ on, either $\operatorname{dist}\left(a_{2 n-1}, \partial^{\dagger} V_{0}\right) \geq \varepsilon$ or $\operatorname{dist}\left(-1-a_{2 n}, \partial^{\dagger} V_{0}\right) \geq \varepsilon$, then $K\left(a_{n} / 1\right)$ converges generally. If $K\left(a_{n} / 1\right)$ is a continued fraction from $\left\langle E_{1}, \widehat{E}_{2, \delta},\right\rangle$ such that for each $n$ from some $n_{0} \in \mathbb{N}$ on, either $\operatorname{dist}\left(-1-a_{2 n+1}, \partial^{\dagger} V_{1}\right) \geq \varepsilon$ or $\operatorname{dist}\left(a_{2 n}, \partial^{\dagger} V_{1}\right) \geq \varepsilon$, then $K\left(a_{n} / 1\right)$ converges generally.

D. Let $K\left(a_{n} / 1\right)$ from $\left\langle E_{1}, E_{2}\right\rangle$ converge generally to $c$. Then $c_{2 n} \rightarrow c$. Let $\varepsilon>0$ and $n_{0} \in \mathbb{N}$. If $-1 \notin V_{0} \backslash\left(-1-V_{1}^{\circ}\right)$ or for each $n \geq n_{0}$ either $\operatorname{dist}\left(a_{2 n-1}, \overline{V_{0}^{c}} \cap\left(-1-V_{1}\right)\right) \geq \varepsilon$ or $d\left(-1-a_{2 n+2}, \overline{V_{0}^{c}}\right) \geq \varepsilon$, then $K\left(a_{n} / 1\right)$ converges to $c$ in the classical sense.

Remarks 2.6.

1. $E_{1}$ is bounded by a cartesian oval with foci at 0 and $\tilde{a}_{1}$. If $C_{1}=-1$, this oval reduces to a circle. $E_{2}$ is an unbounded set.

2. Jones and Thron [6, thm. 5.4], [7, thm. 4.11, p.72], proved the expressions for $E_{1}$ and $E_{2}$ for the case $\left|C_{0}\right|<R_{0} \neq\left|1+C_{0}\right|$ and $\left|1+C_{1}\right|<R_{1} \leq\left|C_{1}\right|$. Theorem 2.5 generalizes their result.

3. This disk - complement of disk case is quite special in the following sense: the case $a /\left(1+V_{k}\right)=V_{k-1}$ does not necessarily occur only for $a \in \partial E_{k}$. Therefore $\widehat{E}_{k, \delta}$ is not necessarily simply connected or even connected. This means that we do not necessarily have that

$\overline{G_{k}} \subseteq E_{k}^{\circ} \quad$ for $k=1,2 \quad \Rightarrow \quad\left\langle G_{1}, G_{2}\right\rangle$ are twin convergence sets as otherwise this is a normal feature for element sets $\langle E, E\rangle$ corresponding to simple value sets $\langle V, V\rangle$. 
The half plane - half plane case.

Let $V_{0}$ and $V_{1}$ be closed half planes,

$$
V_{k}=\left\{z \in \mathbb{C} ; \operatorname{Re}\left(z e^{-i \alpha_{k}}\right) \geq-g_{k} \cos \alpha_{k}\right\} \cup\{\infty\}=H\left(-g_{k} \cos \alpha_{k}, \alpha_{k}\right)
$$

for some $\alpha_{k}, g_{k} \in \mathbb{R}$. Then $E_{k} \neq \emptyset$ only if $0 \in V_{k-1}$, and $-1 \notin V_{k}^{\circ}$. Therefore we require

$$
\left|\alpha_{k}\right| \leq \pi / 2 \quad \text { and } \quad 0 \leq g_{k} \leq 1 \quad \text { for } k=1,2 .
$$

Theorem 2.7. Let $\alpha_{k}, g_{k} \in \mathbb{R}$ satisfy (2.13) and

$$
\left|\alpha_{0}+\alpha_{1}\right|<\pi \text { and } g_{k-1}\left(1-g_{k}\right) \neq 1 \text { for } k=1,2,
$$

and let $K\left(a_{n} / 1\right)$ be a continued fraction from $\left\langle E_{1}, E_{2}\right\rangle$ given by

$$
E_{k}:=\left\{a \in \mathbb{C} ;|a|-\operatorname{Re}\left(a e^{-i\left(\alpha_{0}+\alpha_{1}\right)}\right) \leq 2 g_{k-1}\left(1-g_{k}\right) \cos \alpha_{0} \cos \alpha_{1}\right\} .
$$

Then the even and odd parts of $K\left(a_{n} / 1\right)$ converge to finite values in $V_{0}$, and $K\left(a_{n} / 1\right)$ itself converges if and only if (1.5) holds.

Remarks 2.8.

1. $\left\langle E_{1}, E_{2}\right\rangle$ are the element sets corresponding to $\left\langle V_{0}, V_{1}\right\rangle$ in (2.12). If $g_{k-1}=0$ or if $-1 \in \partial V_{k}$, then $E_{k}$ reduces to the ray $e^{i\left(\alpha_{0}+\alpha_{1}\right)}(0, \infty)$, possibly including the end point $a=0$. (Remember, $0 \notin E_{k}$ if $-1 \in V_{k}$ by definition.)

2. If $E_{k}^{\circ} \neq \emptyset$, then $\partial E_{k}$ is a parabola with axis along the ray

$$
e^{i\left(\alpha_{0}+\alpha_{1}\right)}\left[-g_{k-1}\left(1-g_{k}\right) \cos \alpha_{0} \cos \alpha_{1}, \infty\right)
$$

and focus at the origin.

3. Theorem 2.7 does not contain any essential news compared to the twin version of Jones' and Thron's multiple parabola theorem in [5], 7, thm. 4.43 , p. 106] which says that Theorem 2.7 holds under the additional conditions that $0<g_{k}<1$ and $\left|\alpha_{k}\right|<\pi / 2$ for $k=0$ and $k=1$.

Example 2.9. Let $\alpha_{0}=\alpha_{1}=0, g_{0}=0$ and $g_{1}=1$ in (2.12) and (2.15). Then $0 \in \partial V_{0}$ and $-1 \in \partial V_{1}$; i.e., $-1 \in \partial^{\dagger} V_{1}$. For given positive sequences $\left\{\varepsilon_{n}\right\}$ and $\left\{\delta_{n}\right\}$ converging to 0 , let

$$
t_{2 n-1}:=\varepsilon_{n}-1, \quad t_{2 n}:=\delta_{n} \quad \text { and } \quad a_{n}:=t_{n-1}\left(1+t_{n}\right)
$$

for all $n$. Then $K\left(a_{n} / 1\right)$ is a continued fraction from $\left\langle E_{1}, E_{2}\right\rangle$ given by (2.15). By [12, formula (3.3.3), p.216] it follows that

$$
S_{n}(0)-t_{0}=-\frac{t_{0}}{R_{n}} \quad \text { where } R_{n}:=\sum_{k=0}^{n} P_{k} \text { and } P_{k}:=\prod_{j=1}^{k} \frac{1+t_{j}}{-t_{j}} .
$$

In our situation,

$$
\frac{1+t_{2 n-1}}{-t_{2 n-1}} \cdot \frac{1+t_{2 n}}{-t_{2 n}}=-\frac{\varepsilon_{n}}{1-\varepsilon_{n}} \cdot \frac{1+\delta_{n}}{\delta_{n}} \sim-\frac{\varepsilon_{n}}{\delta_{n}}\left(1+\varepsilon_{n}+\delta_{n}\right),
$$

so $S_{2 n}(0)$ may converge or diverge, depending on the asymptotic behavior of $\left\{\varepsilon_{n}\left(1+\varepsilon_{n}+\delta_{n}\right) / \delta_{n}\right\}$. A similar argument also shows that $K\left(a_{n} / 1\right)$ may also diverge generally in this case. 


\section{Some intermediate ReSUlts}

Let $\left\langle V_{0}, V_{1}\right\rangle$ be closed twin value sets for the continued fraction $K\left(a_{n} / 1\right)$. Then it follows from (1.2) and (1.4) that

$$
\Delta_{n}:=S_{n}\left(V_{n}\right)=S_{n-1} \circ s_{n}\left(V_{n}\right) \subseteq S_{n-1}\left(V_{n-1}\right)=\Delta_{n-1} \subseteq \cdots \subseteq \Delta_{0}=V_{0},
$$

where $V_{2 n}:=V_{0}$ and $V_{2 n+1}:=V_{1}$ for all $n$. Since all $s_{n}$ are (non-singular) linear fractional transformations, so are also $S_{n}$ (see (1.4)). Therefore, since $V_{n}$ is circular, also $\Delta_{n}$ is a circular domain. The nestedness (3.1) implies that $\Delta_{n}$ converges to a limit set $\Delta$. If $\Delta$ just contains one point, the limit point case, then $\left\{S_{2 n}\right\}$ and $\left\{S_{2 n+1}\right\}$ converge uniformly in $V_{0}$ and $V_{1}$ respectively to the limit point $c$. Since both $V_{0}$ and $V_{1}$ contain more than one point in our cases, $K\left(a_{n} / 1\right)$ converges generally to $c$ in this case. If the limit set $\Delta$ has positive or infinite radius, the limit circle case, we need to investigate further. That $\Delta$ is a circular set in this case was proved by Thron [7, thm. 4.2B, p. 66].

In special cases classical convergence to $c$ may be wanted. This may be possible to prove by means of Theorem 1.4. This theorem is partly based on Theorem 3.1 below, which concerns restrained sequences introduced in [4]: we say that a sequence $\left\{F_{n}\right\}$ of linear fractional transformations is restrained if there exist two sequences $\left\{u_{n}\right\}$ and $\left\{v_{n}\right\}$ from $\widehat{\mathbb{C}}$ such that

$$
\lim d\left(F_{n}\left(u_{n}\right), F_{n}\left(v_{n}\right)\right)=0 \text { and } \liminf d\left(u_{n}, v_{n}\right)>0 .
$$

If in addition $\lim F_{n}\left(u_{n}\right)=c$, then we say that $\left\{F_{n}\right\}$ converges generally to $c$. As in (1.9) there exists an exceptional sequence $\left\{z_{n}^{\dagger}\right\}$ for $\left\{F_{n}\right\}$ such that if (3.2) holds, then (see [4])

$$
\lim d\left(F_{n}\left(z_{n}\right), F_{n}\left(u_{n}\right)\right)=0 \quad \text { whenever } \liminf d\left(z_{n}, z_{n}^{\dagger}\right)>0 \text {. }
$$

Theorem 3.1. Let $\left\langle V_{0}, V_{1}\right\rangle$ be closed twin value sets for the continued fraction $K\left(a_{n} / 1\right)$ where $V_{0}$ or $V_{1}$ contains more than one element. Let $k \in\{0,1\}$ be fixed, and let $\left\{S_{2 n+k}\right\}$ be restrained with exceptional sequence $\left\{z_{n}^{\dagger}\right\}$. Then the limit points for $\left\{z_{n}^{\dagger}\right\}$ are contained in $\left(-1-V_{k+1}\right) \backslash V_{k}^{\circ}$, and whenever $\lim \inf d\left(u_{n}, z_{n}^{\dagger}\right)>0$, the set $L$ of the limit points for $S_{2 n+k}\left(u_{n}\right)$ is independent of $\left\{u_{n}\right\}$ and $L \subseteq V_{0} \backslash$ $\left(-1-V_{1}^{\circ}\right)$.

Proof. Since either $V_{0}$ or $V_{1}$ contains at least two points, they both do since $a_{2 n} /\left(1+V_{0}\right) \subseteq V_{1}$ and $a_{2 n+1} /\left(1+V_{1}\right) \subseteq V_{0}$. Since $V_{k}$ contains more than one point, there exists a sequence $\left\{v_{n}\right\}$ from $V_{k}$ with $\lim \inf d\left(v_{n}, z_{n}^{\dagger}\right)>0$. By (3.1) it follows that $S_{2 n+k}\left(V_{k}\right) \subseteq V_{0}$ for all $n$. It follows from (3.3) that $L$ is independent of $\left\{u_{n}\right\}$ when $\liminf d\left(u_{n}, z_{n}^{\dagger}\right)>0$, and thus $L \subseteq V_{0}$. Similarly, by Remark 1.2.2, $L \subseteq W_{0}=-1-\overline{V_{1}^{c}}$, so $L \subseteq V_{0} \cap W_{0}=Y_{0}=V_{0} \backslash\left(-1-V_{1}^{\circ}\right)$.

Evidently, $\left\{z_{n}^{\dagger}\right\}$ can be chosen as $z_{n}^{\dagger}:=S_{2 n+k}^{-1}(p)$ for any $p \notin L$. By (3.3) every exceptional sequence has the same asymptotic behavior. Let $p \notin V_{0}$. Then $z_{n}^{\dagger}:=S_{2 n+k}^{-1}(p) \in V_{k}^{c}$ for all $n$. Similarly, for $q \in W_{k}^{c}$ given by $W_{k}:=\left(-1-\overline{V_{k+1}^{c}}\right)$ we can choose $z_{n}^{\dagger}:=S_{2 n+k}^{-1}(q)$ for all $n$, and then $z_{n}^{\dagger} \in W_{k}^{c}$ for all $n$. (See Remark 1.2.2.) Hence all the limit points of $\left\{z_{n}^{\dagger}\right\}$ are $\subseteq \overline{W_{k}^{c}} \cap \overline{V_{k}^{c}}=\left(-1-V_{k+1}\right) \backslash V_{k}^{\circ}$.

Since $V_{0}$ is a circular domain, there exists a linear fractional transformation $\varphi_{0}$ such that $\varphi_{0}\left(V_{0}\right)=\overline{\mathbb{D}}$. Hence the following result from [10] is useful to establish convergence in the limit circle case. 
Theorem 3.2 ([10, thm. 3.8, 3.10]). Let $\left\{t_{n}\right\}$ be linear fractional transformations with $t_{n}(\mathbb{D}) \subseteq \mathbb{D}$, and let $T_{n}:=t_{1} \circ t_{2} \circ \cdots \circ t_{n}$ for all $n \in \mathbb{N}$. If $R:=\lim \operatorname{rad}\left(T_{n}(\overline{\mathbb{D}})\right)>$ 0 , and there exists a set $I \subseteq \mathbb{N}$ such that

$$
\limsup _{n \in I, n \rightarrow \infty} \operatorname{rad}\left(t_{n}(\partial \mathbb{D})\right)<1 \quad \text { and } \liminf _{n \in \mathbb{N} \backslash I, n \rightarrow \infty} \operatorname{rad}\left(t_{n-1}^{-1}(\partial \mathbb{D})\right)>1,
$$

then $\left|T_{n}^{-1}(\infty)\right| \rightarrow 1$ and $\sum_{n=1}^{\infty}\left|T_{n}^{\prime}(0)\right|<\infty$.

Remarks 3.3.

1. Of course, if $I$ is bounded, then the first condition in (3.4) is void, and if $\mathbb{N} \backslash I$ is bounded, then the second one is void.

2. The conclusion $\sum\left|T_{n}^{\prime}(0)\right|<\infty$ for the derivatives $T_{n}^{\prime}$ implies that $\left\{T_{n}\right\}$ is restrained. (Proof: $T_{n}$ can be written

$$
T_{n}(z)=C_{n}+R_{n} e^{i \omega_{n}} \frac{z-Q_{n}}{1-\bar{Q}_{n} z} \quad \text { for some }\left|Q_{n}\right|<1 \text { and } \omega_{n} \in \mathbb{R}
$$

when $T_{n}(\overline{\mathbb{D}})=B\left(C_{n}, R_{n}\right)$, and thus $T_{n}^{\prime}(z)=R_{n} e^{i \omega_{n}}\left(1-\left|Q_{n}\right|^{2}\right) /\left(1-\bar{Q}_{n} z\right)^{2}$. Hence $T_{n}^{\prime}(z) \rightarrow 0$ for all $z \in \mathbb{D}$.) Indeed, $\sum\left|T_{n}^{\prime}(z)\right|<\infty$ for every $z \in \mathbb{D}$.

Let $\mathcal{M}$ be the family of (non-singular) linear fractional transformations. For given $V \subseteq \widehat{\mathbb{C}}$ and $\varepsilon>0$ we introduced the subfamily

$$
\mathcal{M}_{\varepsilon}(V):=\left\{t \in \mathcal{M} ; t(V) \subseteq V \backslash B_{d}(z, \varepsilon) \text { for some } z \in \partial V\right\}
$$

in [11. This notation is useful to convert Theorem 3.2 to our situation:

Corollary 3.4. Let $k \in\{0,1\}$ be fixed, and let $\left\langle V_{0}, V_{1}\right\rangle$ be closed circular twin value sets for the continued fraction $K\left(a_{n} / 1\right)$ where the limit circle case occurs. Furthermore, let $\sigma_{n}:=s_{2 n-1+k} \circ s_{2 n+k}, \sigma_{0}:=\sigma_{1}$ and assume that

$$
\sigma_{n} \in \mathcal{M}_{\varepsilon}\left(V_{k}\right) \text { for all } n \in I \quad \text { and } \quad \sigma_{n-1}^{-1} \in \mathcal{M}_{\varepsilon}\left(V_{k}^{c}\right) \text { for all } n \in \mathbb{N} \backslash I
$$

for some $I \subseteq \mathbb{N}$ and $\varepsilon>0$. Then $\left\{S_{2 n+k}\right\}$ is restrained and its exceptional sequences $\left\{z_{n}^{\dagger}\right\}$ have all their limit points $\in \partial V_{k}$. If also $V_{0}$ is bounded, then $\left\{\zeta_{2 n+k}\right\}$ is an exceptional sequence for $\left\{S_{2 n+k}\right\}$ and $\sum_{n=1}^{\infty}\left|S_{2 n+k}^{\prime}(z)\right|<\infty$ for every finite $z \in V_{k}^{\circ}$.

Proof. Let $\varphi \in \mathcal{M}$ satisfy $\varphi\left(V_{k}\right)=\overline{\mathbb{D}}$. Then $t_{n}:=\varphi \circ \sigma_{n} \circ \varphi^{-1}$ maps $\mathbb{D}$ into $\mathbb{D}$, and $T_{n}:=t_{1} \circ t_{2} \circ \cdots \circ t_{n}=\varphi \circ S_{2 n}^{(k)} \circ \varphi^{-1}$ where $S_{2 n}^{(k)}:=\sigma_{1} \circ \sigma_{2} \circ \cdots \circ \sigma_{n}$. Condition (3.6) implies (3.4). Hence $\left\{T_{n}\right\}$ is restrained with exceptional sequence $\left\{T_{n}^{-1}(\infty)\right\}$ where $\left|T_{n}^{-1}(\infty)\right| \rightarrow 1$. Therefore $\left\{S_{2 n}^{(k)}\right\}$ is restrained with exceptional sequence $z_{n}^{\dagger}:=$ $\varphi^{-1} \circ T_{n}^{-1}(\infty)=\left(S_{2 n}^{(k)}\right)^{-1}\left(\varphi^{-1}(\infty)\right)$. That $\left\{S_{2 n+k}\right\}$ is restrained with exceptional sequence $\left\{z_{n}^{\dagger}\right\}$ follows therefore since $S_{2 n}=S_{2 n}^{(0)}$ and $S_{2 n+1}=s_{1} \circ S_{2 n}^{(1)}$ for the fixed $s_{1} \in \mathcal{M}$. Since $\left|T_{n}^{-1}(\infty)\right| \rightarrow 1$, i.e., $\operatorname{dist}\left(T_{n}^{-1}(\infty), \partial \mathbb{D}\right) \rightarrow 0$, it follows that $d\left(\varphi^{-1} \circ T_{n}^{-1}(\infty), \varphi^{-1}(\partial \mathbb{D})\right) \rightarrow 0$ where $\varphi^{-1}(\partial \mathbb{D})=\partial V_{k}$ and $\varphi^{-1} \circ T_{n}^{-1}(\infty)=z_{n}^{\dagger}$. That is, all the limit points of $\left\{z_{n}^{\dagger}\right\}$ are $\in \partial V_{k}$.

Let $V_{0}$ be bounded. Then $\infty \notin V_{0}$, so $\left\{\zeta_{2 n+k}\right\}$ is an exceptional sequence for $\left\{S_{2 n+k}\right\}$ since $S_{2 n+k}\left(\zeta_{2 n+k}\right)=\infty$ whereas all the limit points for $\left\{S_{2 n+k}\left(u_{n}\right)\right\}$ are $\in V_{0}$ when $\liminf d\left(u_{n}, z_{n}^{\dagger}\right)>0$ (Theorem 3.1). It remains to prove that $\sum\left|S_{2 n+k}^{\prime}(z)\right|<\infty$ for finite $z \in V_{k}^{\circ}$. By Theorem 3.2 and Remark 3.3.2 we know that $\sum\left|T_{n}^{\prime}(w)\right|<\infty$ for every $w \in \mathbb{D}$. First let $k=0$ and choose $\varphi(z):=$ $\left(z-C_{0}\right) / R_{0}$ where $C_{0}$ and $R_{0}$ are the center and radius of $V_{0}$. Let $z \in V_{0}^{\circ}$ be arbitrarily chosen, and let $w:=\varphi(z)$. Then $w \in \mathbb{D}$ and $S_{2 n}^{\prime}(z)=\left(\varphi^{-1}\right)^{\prime}\left(T_{n}(\varphi(z))\right)$. 
$T_{n}^{\prime}(\varphi(z)) \cdot \varphi^{\prime}(z)=\left(\varphi^{-1}\right)^{\prime}\left(T_{n}(w)\right) \cdot T_{n}^{\prime}(w) \cdot \frac{1}{R_{0}}=R_{0} \cdot T_{n}^{\prime}(w) \cdot \frac{1}{R_{0}}=T_{n}^{\prime}(w)$. Hence $\sum\left|S_{2 n}^{\prime}(z)\right|<\infty$.

Next let $k=1$ and set $\widehat{V}_{0}:=s_{1}\left(V_{1}\right)$. Then $\widehat{V}_{0}=B\left(\widehat{C}_{0}, \widehat{R}_{0}\right) \subseteq V_{0}$ for some fixed $\widehat{C}_{0} \in \mathbb{C}$ and $\widehat{R}_{0}>0$. Furthermore, let $\varphi_{1}(z):=\left(z-\widehat{C}_{0}\right) / \widehat{R}_{0}$ so that $\varphi_{1}\left(\widehat{V}_{0}\right)=\overline{\mathbb{D}}$ and $t_{n}:=\varphi_{1} \circ s_{1} \circ s_{2 n} \circ s_{2 n+1} \circ s_{1}^{-1} \circ \varphi_{1}^{-1}$ maps $\mathbb{D}$ into $\mathbb{D}$. Let a finite $z \in V_{1}^{\circ}$ be arbitrarily chosen, and let $w:=\varphi_{1} \circ s_{1}(z)$. Then $w \in \mathbb{D}$ and

$$
\begin{aligned}
S_{2 n+1}^{\prime}(z) & =\left(\varphi_{1}^{-1}\right)^{\prime}\left(T_{n} \circ \varphi_{1} \circ s_{1}(z)\right) \cdot T_{n}^{\prime}\left(\varphi_{1} \circ s_{1}(z)\right) \cdot \varphi_{1}^{\prime}\left(s_{1}(z)\right) \cdot s_{1}^{\prime}(z) \\
& =\widehat{R}_{0} \cdot T_{n}^{\prime}(w) \cdot \frac{1}{\widehat{R}_{0}} \cdot \frac{-a_{1}}{(1+z)^{2}}=\frac{-a_{1}}{(1+z)^{2}} T_{n}^{\prime}(w)
\end{aligned}
$$

where $z \neq-1$ since $-1 \notin V_{1}$ when $V_{0}$ is bounded. Hence $\sum\left|S_{2 n+1}^{\prime}(z)\right|<\infty$.

It follows from (1.6) that $S_{n}$ can be written

$$
S_{n}(z)=\left\{\begin{array}{lr}
c_{n-1}-\frac{\zeta_{n}\left(c_{n}-c_{n-1}\right)}{z-\zeta_{n}} & \text { if } \zeta_{n} \neq \infty, \\
c_{n}-\left(c_{n-2}-c_{n}\right) z & \text { if } \zeta_{n}=\infty .
\end{array}\right.
$$

Therefore

$$
S_{n}^{\prime}(z)=\left\{\begin{array}{l}
\frac{\zeta_{n}\left(c_{n}-c_{n-1}\right)}{\left(z-\zeta_{n}\right)^{2}}=-\frac{S_{n}(z)-c_{n-1}}{z-\zeta_{n}} \text { if } \zeta_{n} \neq \infty \\
c_{n}-c_{n-2} \quad \text { if } \zeta_{n}=\infty .
\end{array}\right.
$$

Under the conditions of Corollary 3.4 it follows therefore that for arbitrary $\varepsilon>0$,

$$
\begin{aligned}
& \sum^{\prime}\left|S_{2 n+k}\left(z_{n}\right)-c_{2 n+k-1}\right|<\infty \\
& \text { whenever } \varepsilon \leq \operatorname{dist}\left(z_{n}, Z_{k}\right) \leq \frac{1}{\varepsilon} \text { for all } n \text { and } \infty \notin Z_{k} .
\end{aligned}
$$

(For the notation $\sum^{\prime}$ and $Z_{k}$, see the list of notation in section 1.) This leads to the following result, where $W_{k}:=-1-\overline{V_{k+1}^{c}}$ and $\partial^{*} V_{k}:=\partial V_{k} \cap\left(-1-V_{k+1}\right)$ as usual.

Theorem 3.5. Let $k \in\{0,1\}$ be fixed. Let $\left\langle V_{0}, V_{1}\right\rangle$ be closed circular twin value sets for the continued fraction $K\left(a_{n} / 1\right)$ where $V_{0}$ is bounded, the limit circle case occurs and (3.6) holds for our $k$ for some $I \subseteq \mathbb{N}$ and $\varepsilon>0$. Then $Z_{k} \subseteq \partial^{*} V_{k}$, $-k \notin Z_{k}, 0 \notin Z_{0}$ and $Z_{1}$ and $Z_{k}$ are bounded, $\sum^{\prime}\left|c_{2 n}-c_{2 n-1}\right|<\infty$, and the following statements are true.

A. Let $\varepsilon>0$. If $(k-1) \notin Z_{k}$ or if for each $n$ from some $n_{0}$ on, either $\operatorname{dist}\left(a_{2 n+k-1}, Z_{k}\right) \geq \varepsilon$ or $\operatorname{dist}\left(-1-a_{2 n+k}, Z_{k}\right) \geq \varepsilon$, then $\sum^{\prime}\left|c_{n}-c_{n-1}\right|<\infty$.

B. If also the limit circle case occurs for $S_{2 n}\left(W_{0}\right)$ and

$$
\sigma_{n} \in \mathcal{M}_{\varepsilon}\left(W_{k}\right) \text { for } n \in I \quad \text { and } \quad \sigma_{n-1}^{-1} \in \mathcal{M}_{\varepsilon}\left(W_{k}^{c}\right) \text { for } n \in \mathbb{N} \backslash I
$$

for some $I \subseteq \mathbb{N}$ and $\varepsilon>0$ for $\sigma_{n}$ as in Corollary 3.4, then $Z_{k} \subseteq \partial^{\dagger} V_{k}$.

Proof. Under our conditions, $\left\{S_{2 n+k}\right\}$ is restrained with exceptional sequence $\left\{\zeta_{2 n+k}\right\}, Z_{k} \subseteq\left(-1-V_{k+1}\right) \cap \partial V_{k}=\partial^{*} V_{k}$ and $Z_{k+1} \subseteq\left(-1-V_{k}\right) \backslash V_{k+1}^{\circ}$ (Theorem 3.1 and Corollary 3.4). Now, $V_{0}$ is bounded, so $-1 \notin V_{1}$, and thus $0 \notin Z_{0}$ and $-k \notin Z_{k}$, and $Z_{k}$ and $Z_{1}$ are bounded. Since $S_{2 n}(0)=c_{2 n}$ and $S_{2 n+1}(-1)=c_{2 n-1}$, it follows therefore from (3.9) that $\sum^{\prime}\left|c_{2 n}-c_{2 n-1}\right|<\infty$.

A. It suffices to prove that either $\sum^{\prime}\left|c_{2 n-2}-c_{2 n-1}\right|<\infty$ or $\sum^{\prime} \mid c_{2 n+m}-$ $c_{2 n+m-2} \mid<\infty$ for an $m \in\{0,1\}$. First let $(k-1) \notin Z_{k}$. If $k=0$, this means that 
$\sum^{\prime}\left|S_{2 n}(-1)-c_{2 n-1}\right|<\infty$ by (3.9) where $S_{2 n}(-1)=c_{2 n-2}$. If $k=1$, then $0 \notin Z_{1}$ and $\sum^{\prime}\left|S_{2 n+1}(0)-c_{2 n}\right|<\infty$. Next let $I:=\left\{n \in \mathbb{N} ; \operatorname{dist}\left(a_{2 n+k-1}, Z_{k}\right) \geq \varepsilon\right\}$. Then $\sum_{n \in I}^{\prime}\left|S_{2 n+k-2}\left(a_{2 n+k-1}\right)-c_{2 n+k-3}\right|<\infty$ where $S_{2 n+k-2}\left(a_{2 n+k-1}\right)=c_{2 n+k-1}$ and $\sum_{n \notin I}^{\prime}\left|S_{2 n+k}\left(-1-a_{2 n+k}\right)-c_{2 n+k-1}\right|<\infty$ where $S_{2 n+k}\left(-1-a_{2 n+k}\right)=c_{2 n+k-3}$, which means that $\sum^{\prime}\left|c_{2 n+k+1}-c_{2 n+k-1}\right|<\infty$.

B. $\left\langle W_{0}, W_{1}\right\rangle$ are twin value sets for $K\left(a_{n} / 1\right)$ (Remark 1.2.2). They satisfy the conditions in Corollary 3.4, so the exceptional sequences for $\left\{S_{2 n+k}\right\}$ have all their limit points in $\partial W_{k}$. Hence $Z_{k} \subseteq \partial V_{k} \cap \partial W_{k}=\partial V_{k} \cap\left(-1-\partial V_{k+1}\right)=\partial^{\dagger} V_{k}$.

\section{Proofs}

Inspired by (3.5) we define

$$
\begin{gathered}
\mathcal{M}_{\varepsilon}(V, W):=\left\{t \in \mathcal{M} ; t(V) \subseteq W \backslash B_{d}(z, \varepsilon) \text { for some } z \in \partial W\right\}, \\
\mathcal{E}(V):=\left\{\langle A, B\rangle \subseteq \mathbb{C}^{2} ;\right. \\
\left.\exists \varepsilon>0 \text { s.t. } s_{1} \circ s_{2} \in \mathcal{M}_{\varepsilon}(V) \text { for all }\left\langle a_{1}, a_{2}\right\rangle \in\langle A, B\rangle\right\}, \\
\mathcal{E}(V, W):=\left\{A \subseteq \mathbb{C} ; \exists \varepsilon>0 \text { s.t. } s \in \mathcal{M}_{\varepsilon}(V, W) \text { for all } a \in A\right\} .
\end{gathered}
$$

Proof of Theorem 1.4. Since $K\left(a_{n} / 1\right)$ converges generally to $c$ whereas $q \neq c$, the sequence $\left\{S_{n}\right\}$ is restrained with exceptional sequence $z_{n}^{\dagger}:=S_{n}^{-1}(q)$. Part A follows from Theorem 3.1. The result in B follows from (1.9) since $S_{2 n+k}(-1)=$ $c_{2 n+k-2}$ and $S_{2 n+k}(\infty)=c_{2 n+k-1}$. Similarly, part C follows from (1.9) since $S_{2 n+k-2}\left(a_{2 n+k-1}\right)=c_{2 n+k-1}$ and $S_{2 n+k+2}\left(-1-a_{2 n+k+2}\right)=c_{2 n+k-1}$.

To prove part $\mathrm{D}$ we observe that if $V_{0}$ is bounded, then $c \neq \infty$ and $\infty \notin \tilde{Z}_{1}$ by part A. Hence $\left\{\zeta_{n}\right\}$ is exceptional and $S_{2 n+1}(\infty)=c_{2 n} \rightarrow c$ by part B. Finally, if $-1 \notin V_{0} \backslash\left(-1-V_{1}^{\circ}\right)$, i.e., $0 \notin\left(-1-V_{0}\right) \backslash V_{1}^{\circ}$, then $0 \notin \tilde{Z}_{1}$, and part $\mathrm{E}$ follows from part B. (The same holds true if $0 \notin V_{0}$, but $0 \notin V_{0} \Rightarrow \infty \notin V_{1} \Rightarrow-1 \notin V_{0}$.)

Lemma 4.1. For given closed twin value sets $\left\langle V_{0}, V_{1}\right\rangle$, let $U_{k}:=-1-V_{k+1}^{c}$ for $k=0,1$, and let $k \in\{0,1\}$ be a fixed number. Then $s\left(U_{k}\right) \subseteq U_{k+1}$ if and only if $s\left(V_{k}\right) \subseteq V_{k+1}$ and $s\left(U_{k}\right)=U_{k+1}$ if and only if $s\left(V_{k}\right)=V_{k+1}$. Similarly, if $A \subseteq \mathbb{C}$ is a closed set with $0, \infty \notin A$, then $A \in \mathcal{E}\left(U_{k}, U_{k+1}\right)$ if and only if $A \in \mathcal{E}\left(V_{k}, V_{k+1}\right)$.

Proof. Let $a /\left(1+V_{k}\right) \subseteq V_{k+1}$. Since $V_{k}$ is closed, the set $V_{k}^{c}$ is open and non-empty, and both $V_{k}, V_{k+1}, U_{k}$ and $U_{k+1}$ contain finite elements. Therefore

$$
\frac{a}{1+U_{k}}=-\frac{a}{V_{k+1}^{c}}=-\left(\frac{a}{V_{k+1}}\right)^{c} \subseteq\left(-1-V_{k}\right)^{c}=U_{k+1} .
$$

This actually proves the first two equivalences since $U$ and $V$ can be interchanged in this inclusion. Let $a /\left(1+V_{k}\right) \subseteq V_{k+1} \backslash B_{d}(z, \varepsilon)$ for some finite $z \in \partial V_{k+1}=-1-\partial U_{k}$ and $\varepsilon>0$. That is, $a / U_{k+1} \supseteq-\left(V_{k+1} \backslash B_{d}(z, \varepsilon)\right)^{c}=-\left(-1-U_{k}\right) \cup B_{d}(z, \varepsilon)=$ $1+U_{k} \cup B_{d}\left(z^{*}, \varepsilon\right)$ where $z^{*}:=-1-z \in \partial U_{k}$. That is, $s^{-1}\left(U_{k+1}\right) \supseteq U_{k} \cup B_{d}\left(z^{*}, \varepsilon\right)$, so $s\left(U_{k} \cup B_{d}\left(z^{*}, \varepsilon\right)\right) \subseteq U_{k+1}$. Let $D:=B_{d}\left(z^{*}, \varepsilon\right) \backslash U_{k}$ so that $U_{k} \cap D=\emptyset$ and $U_{k} \cup D=U_{k} \cup B_{d}\left(z^{*}, \varepsilon\right)$. Then

$$
\frac{a}{1+U_{k} \cup B_{d}\left(z^{*}, \varepsilon\right)}=\frac{a}{1+U_{k}} \cup \frac{a}{1+D} \subseteq U_{k+1} ; \quad \text { i.e., } \quad \frac{a}{1+U_{k}} \subseteq U_{k+1} \backslash \frac{a}{1+D}
$$

where $a /\left(1+z^{*}\right) \in U_{k+1}$. Therefore $a /\left(1+U_{k}\right) \subseteq U_{k+1} \backslash B_{d}\left(\frac{a}{1+z^{*}}, \varepsilon^{*}\right)$ where $\varepsilon^{*}:=\operatorname{dist}\left(\frac{a}{1+z^{*}}, \frac{a}{1+\partial B_{d}(z, \varepsilon)}\right)$. Since $0, \infty \notin A$, the quantity $\varepsilon^{*}$ has a positive lower bound for $a \in A$. Therefore $A \in \mathcal{E}\left(U_{k}, U_{k+1}\right)$. This proves the last equivalence. 
Lemma 4.2. Let $V_{0}, V_{1}$ be closed circular domains, and let $a_{1}, a_{2} \in \mathbb{C} \backslash\{0\}$ satisfy $a_{k} /\left(1+V_{k}\right) \subseteq V_{k-1}$ for $k=1,2$. Then $\sigma:=s_{1} \circ s_{2}$ is an elliptic transformation if and only if $s_{k}\left(V_{k}\right)=V_{k-1}$ for $k=1,2$ and $\sigma$ has exactly two distinct fixed points $w_{0}, w_{1} \notin \partial V_{0}$.

Proof. Let $\sigma$ be elliptic. Since $\sigma\left(V_{0}\right) \subseteq V_{0}$, it follows from [11, thm. 1.4] that $\sigma\left(V_{0}\right)=V_{0}$. Since $V_{0}=s_{1} \circ s_{2}\left(V_{0}\right) \subseteq s_{1}\left(V_{1}\right) \subseteq V_{0}$, this means that $s_{1}\left(V_{1}\right)=V_{0}$ and $s_{2}\left(V_{0}\right)=V_{1}$. It is clear that $\sigma$ has two distinct fixed points $w_{0}, w_{1}$ and that $\partial V_{0}$ is a fixed circle (or fixed line) for $\sigma$. Hence $\partial V_{0}$ separates the two fixed points.

Conversely, assume that $s_{k}\left(V_{k}\right)=V_{k-1}$ for $k=1,2$ and that $\sigma$ has two distinct fixed points $\notin \partial V_{0}$. Then $\sigma\left(\partial V_{0}\right)=\partial V_{0}$, which means that $\sigma$ is either hyperbolic, parabolic, elliptic or the identity transformation. Since $\sigma$ has exactly two distinct fixed points, the parabolic case and the identity case are ruled out. Since none of the fixed points lie on $\partial V_{0}$, the hyperbolic case is ruled out, so $\sigma$ is elliptic.

Lemma 4.3 (The disk - disk case). Let $V_{k}:=B\left(C_{k}, R_{k}\right)$ for $k=0,1$, where $C_{k} \in \mathbb{C}$ and $R_{k}>0$ satisfy (2.1). Then $\left\langle E_{1}, E_{2}\right\rangle$ given by (2.4) are the corresponding element sets. Let $k \in\{1,2\}$ be fixed. Then $E_{k} \neq \emptyset$ if and only if (2.2) holds. If (2.2) holds with strict inequality, then $E_{k}^{\circ} \neq \emptyset$. If (2.2) holds with equality, then $E_{k}=\left\{\tilde{a}_{k}\right\}$ is given by (2.3) and $\tilde{a}_{k} \neq 0$. If $E_{k}^{\circ} \neq \emptyset$, then $\left\langle E_{k}, E_{k+1}\right\rangle \in \mathcal{E}\left(V_{k-1}\right)$.

Proof. For fixed $k \in\{1,2\}$ and $a \neq 0$ we have

$$
\frac{a}{1+V_{k}}=B\left(\frac{a\left(1+\bar{C}_{k}\right)}{\left|1+C_{k}\right|^{2}-R_{k}^{2}}, \frac{|a| R_{k}}{\left|1+C_{k}\right|^{2}-R_{k}^{2}}\right)=: B\left(\widehat{C}_{k-1}, \widehat{R}_{k-1}\right)
$$

and $a /\left(1+V_{k}\right) \subseteq V_{k-1}$ if and only if $\left|\widehat{C}_{k-1}-C_{k-1}\right|+\widehat{R}_{k-1} \leq R_{k-1}$, that is, if and only if $a \in E_{k}$, where $E_{k}$ is given by (2.4). Since $R_{k}<\left|1+C_{k}\right|$, we see from (2.4) that $E_{k} \neq \emptyset$ if and only if $\tilde{a}_{k} \in E_{k}$, which proves that (2.2) is necessary and sufficient. It also proves that $\tilde{a}_{k}$ is the only point in $E_{k}$ if and only if (2.2) holds with equality, and that $E_{k}^{\circ} \neq \emptyset$ otherwise. This means that if $E_{k}^{\circ} \neq \emptyset$, then $s \in \mathcal{M}_{\varepsilon_{a}}\left(V_{k}, V_{k-1}\right)$ for some $\varepsilon_{a}>0$ for every $a \in E_{k}$. Since $E_{k}$ is compact in $\mathbb{C}\left(-1 \notin V_{k}\right.$ when $V_{k-1}$ is bounded $)$, this means that $E_{k} \in \mathcal{E}\left(V_{k}, V_{k-1}\right)$. Finally, since $s_{k} \circ s_{k+1}\left(V_{k+1}\right) \subseteq s_{k}\left(V_{k}\right)$ for all $\left\langle a_{k}, a_{k+1}\right\rangle \in\left\langle E_{k}, E_{k+1}\right\rangle$, it follows that $\left\langle E_{k}, E_{k+1}\right\rangle \in \mathcal{E}\left(V_{k-1}\right)$.

Proof of Theorem 2.1. If $\left|C_{k-1}\right| R_{k}=\left|1+C_{k}\right| R_{k-1}$ for $k=1$ and $k=2$, then $K\left(a_{n} / 1\right)$ with all $a_{2 n-1}=\tilde{a}_{1}$ and $a_{2 n}=\tilde{a}_{2}$ is the only continued fraction from $\left\langle E_{1}, E_{2}\right\rangle$. It converges if and only if $\tilde{s}_{1} \circ \tilde{s}_{2}$ is non-elliptic. Let (2.2) hold with strict inequality for at least one $k \in\{1,2\}$. Without loss of generality we assume that $E_{1}^{\circ} \neq \emptyset$. (See Remark 1.2.1.)

Assume first that the limit point case occurs. Then $K\left(a_{n} / 1\right)$ converges generally to a value $c \in V_{0}$. It follows by Lemma $1.4 \mathrm{D}$ that $c_{2 n} \rightarrow c$. Since also $V_{1}$ is bounded, we have $-1 \notin V_{0}$, so also $c_{2 n+1} \rightarrow c$ by Lemma $1.4 \mathrm{E}$.

Assume next that the limit circle case occurs. By Lemma 4.3 we know that limsup $\operatorname{rad}\left(s_{2 n-1} \circ s_{2 n}\left(V_{0}\right)\right)<\operatorname{rad}\left(V_{0}\right)$, and so $Z_{0} \subseteq \partial^{*} V_{0}$ by Theorem 3.5. Now, $-1 \notin V_{0}$ implies that $-1 \notin \partial^{*} V_{0}$. Hence $\sum^{\prime}\left|c_{n}-c_{n-1}\right|<\infty$ by Theorem 3.5A, and thus $K\left(a_{n} / 1\right)$ converges.

Lemma 4.4 (The disk - half plane case). Let $V_{0}:=B\left(C_{0}, R_{0}\right)$ and $V_{1}:=$ $H(h \cos \alpha, \alpha)$ where $C_{0} \in \mathbb{C}$ and $R_{0}, h, \alpha \in \mathbb{R}$ satisfy (2.5). Then $\left\langle E_{1}, E_{2}\right\rangle$ given by 
(2.7) are the corresponding element sets, and $\tilde{E}_{k, \delta}$ given by (2.8) satisfies $\tilde{E}_{k, \delta} \in$ $\mathcal{E}\left(V_{k}, V_{k-1}\right)$ for $k=1,2$ and $0<\delta<\left|a_{1}^{*}\right|$.

Proof. For $a \neq 0$ we have

$$
\frac{a}{1+V_{1}}=B\left(\frac{a e^{-i \alpha}}{2(1+h) \cos \alpha}, \frac{|a|}{2(1+h) \cos \alpha}\right)
$$

which is $\subseteq V_{0}$ if and only if $\left|\frac{a e^{-i \alpha}}{2(1+h) \cos \alpha}-C_{0}\right|+\frac{|a|}{2(1+h) \cos \alpha} \leq R_{0}$, i.e., if and only if $a \in E_{1}$. Since $0 \in V_{0}$ and $0 /\left(1+V_{1}\right)=\{0\}$, we also have $0 \in E_{1}$. Similarly, for $a \neq 0$,

$$
\frac{a}{1+V_{0}}= \begin{cases}B\left(\frac{a\left(1+\overline{C_{0}}\right)}{\left|1+C_{0}\right|^{2}-R_{0}^{2}}, \frac{|a| R_{0}}{\left|1+C_{0}\right|^{2}-R_{0}^{2}}\right)=: B\left(\widehat{C}_{1}, \widehat{R}_{1}\right) & \text { if }\left|1+C_{0}\right|>R_{0}, \\ H\left(|a| /\left(2 R_{0}\right), \arg \left(a\left(1+\overline{C_{0}}\right)\right)\right) & \text { if }\left|1+C_{0}\right|=R_{0},\end{cases}
$$

and thus $a /\left(1+V_{0}\right) \subseteq V_{1}$ if and only if

$$
\begin{array}{r}
\operatorname{Re}\left(\frac{a\left(1+\overline{C_{0}}\right)}{\left|1+C_{0}\right|^{2}-R_{0}^{2}} e^{-i \alpha}\right)-\frac{|a| R_{0}}{\left|1+C_{0}\right|^{2}-R_{0}^{2}} \geq h \cos \alpha \quad \text { if }\left|1+C_{0}\right|>R_{0}, \\
\arg \left(a\left(1+\overline{C_{0}}\right)\right)=\alpha \quad \text { and } \quad \frac{|a|}{2 R_{0}} \geq h \cos \alpha \quad \text { if }\left|1+C_{0}\right|=R_{0},
\end{array}
$$

i.e., if and only if $a \in E_{2}$. If $-1 \in V_{0}$, i.e., $\left|1+C_{0}\right|=R_{0}$, then $0 \notin E_{2}$ by definition. Hence $\left\langle E_{1}, E_{2}\right\rangle$ are the element sets corresponding to $\left\langle V_{0}, V_{1}\right\rangle$.

By (4.5) it follows that $a /\left(1+V_{1}\right)=V_{0}$ if and only if $R_{0}=\left|C_{0}\right|$ and $C_{0}=$ $a e^{-i \alpha} /[2(1+h) \cos \alpha]$, i.e., $a=a_{1}^{*}$. Since $-1 \notin V_{1}$, the set $E_{1}$ is compact, so this shows that $E_{1} \in \mathcal{E}\left(V_{1}, V_{0}\right)$ if $R_{0}>\left|C_{0}\right|$. Let $R_{0}=\left|C_{0}\right|$. Since $\tilde{E}_{1, \delta} \subseteq E_{1}$ is a compact set not containing $a_{1}^{*}, \tilde{E}_{1, \delta} \in \mathcal{E}\left(V_{1}, V_{0}\right)$.

Next we study $\tilde{E}_{2, \delta}$. First let $\left|1+C_{0}\right|=R_{0}$. By (4.6) it follows that $a /\left(1+V_{0}\right)=$ $V_{1}$ for $a \neq 0$ if and only if $h>0$ and $q:=\frac{a}{2 R_{0}} \frac{1+\overline{C_{0}}}{\left|1+C_{0}\right|}=h e^{i \alpha} \cos \alpha$, i.e., $a=a_{2}^{*}$. In this case $a_{2}^{*} \neq 0$ and $E_{2}$ is the ray $E_{2}=a_{2}^{*}[1, \infty)$ and $\tilde{E}_{2, \delta}=a_{2}^{*}\left[1+\delta /\left|a_{2}^{*}\right|, \infty\right)$. Hence $\tilde{E}_{2, \delta}$ is a closed set in $\mathbb{C}$ with $0 \notin \tilde{E}_{2, \delta}$, and even if $a_{2 n_{m}} \rightarrow \infty$ as $m \rightarrow \infty$, the set $a_{2 n_{m}} /\left(1+V_{0}\right)$ will not approach $V_{1}$. (Indeed, it approaches the point set $\{\infty\}$ since $V_{0}$ is bounded.) Therefore $\tilde{E}_{2, \delta} \in \mathcal{E}\left(V_{0}, V_{1}\right)$ if $h>0$. If $h<0$, then $\operatorname{dist}\left(q-h e^{i \alpha} \cos \alpha\right)>|h| \cos \alpha>0$, and $E_{2} \in \mathcal{E}\left(V_{0}, V_{1}\right)$. If $h=0$, then $\tilde{E}_{2, \delta}=[\delta, \infty) e^{i \gamma}$ with $\gamma:=\alpha+\arg \left(1+C_{0}\right)$, and $a /\left(1+V_{0}\right)$ is the half plane $H\left(|a| / 2 R_{0}, \arg \left(a\left(1+\overline{C_{0}}\right)\right)\right)=H\left(|a| / 2 R_{0}, \alpha\right)$ for $a \in E_{2}$. Hence also now $\tilde{E}_{2, \delta} \in$ $\mathcal{E}\left(V_{0}, V_{1}\right)$.

Next let $\left|1+C_{0}\right|>R_{0}$. Then it follows from (4.6) that $a /\left(1+V_{0}\right)=B\left(\widehat{C}_{1}, \widehat{R}_{1}\right)$ is a disk not containing the origin for $a \neq 0$. If $a=0$, then $a /\left(1+V_{0}\right)=\{0\}$ since $-1 \notin V_{0}$. Hence, there is no possibility of $B\left(\widehat{C}_{1}, \widehat{R}_{1}\right) \rightarrow V_{1}$ unless $\widehat{R}_{1} \rightarrow \infty$; i.e., $|a| \rightarrow \infty$, but then $a /\left(1+V_{0}\right) \rightarrow\{\infty\}$ since $V_{0}$ is bounded. Hence $E_{2} \in \mathcal{E}\left(V_{0}, V_{1}\right)$ in this case.

Proof of Theorem 2.3. Let $K\left(a_{n} / 1\right)$ be a continued fraction from $\left\langle E_{1}, E_{2}\right\rangle$. If $\operatorname{rad}\left(S_{2 n}\left(V_{0}\right)\right) \rightarrow 0$ or $\operatorname{rad}\left(S_{2 n}\left(W_{0}\right)\right) \rightarrow 0$ or $\operatorname{diam}\left(S_{2 n}\left(Y_{0}\right)\right) \rightarrow 0$, then $K\left(a_{n} / 1\right)$ clearly converges generally. Assume in the proof of parts $\mathrm{A}-\mathrm{C}$ below that $\operatorname{diam}\left(S_{2 n}\left(Y_{0}\right)\right) \rightarrow \tilde{d}>0$, and thus $\operatorname{rad}\left(S_{2 n}\left(V_{0}\right)\right) \rightarrow R>0$ and $\operatorname{rad}\left(S_{2 n}\left(W_{0}\right)\right) \rightarrow$ $R^{*}>0$. 
A. Let $K\left(a_{n} / 1\right)$ be from $\left\langle E_{1}, \tilde{E}_{2, \delta}\right\rangle$. Then $s_{2 n} \circ s_{2 n+1}\left(V_{1}\right) \subseteq s_{2 n}\left(V_{0}\right)$ where $a_{2 n} \in \tilde{E}_{2, \delta} \in \mathcal{E}\left(V_{0}, V_{1}\right)$ by Lemma 4.4 , so $\left\langle\tilde{E}_{2, \delta}, E_{1}\right\rangle \in \mathcal{E}\left(V_{1}\right)$. Therefore $K\left(a_{n} / 1\right)$ converges in the classical sense if $0 \notin Z_{1}$ (Theorem $3.5 \mathrm{~A}$ with $k=1$ ).

Let $0 \in Z_{1}$. Since by Theorem 3.5, $Z_{1} \subseteq \partial^{*} V_{1}$, this means that $0 \in \partial V_{1}$, and $-1 \in V_{0}$, which means that $-1 \in \partial V_{0}$ by $(2.5)$, so indeed, $0 \in \partial^{\dagger} V_{1}$. Then $h=0$, and thus $a_{2}^{*}=0$, and $R_{0}=\left|1+C_{0}\right|$ and $\tilde{E}_{2, \delta}=e^{i \gamma}[\delta, \infty)$ where $\gamma:=\alpha+\arg \left(1+C_{0}\right)$. This means that $\operatorname{dist}\left(\tilde{E}_{2, \delta}, \partial^{*} V_{1}\right)>0$ unless $\tilde{E}_{2, \delta} \subseteq \partial V_{1}$. Now, $\operatorname{Re}\left(C_{0}\right) \geq-\frac{1}{2}$ when $-1 \in \partial V_{0}$ since $0 \in V_{0}$ by (2.5) and $V_{0}$ is a disk. Therefore $\gamma \neq \alpha \pm \frac{\pi}{2}$, and $\tilde{E}_{2, \delta} \not \subset \partial V_{1}$. Hence $K\left(a_{n} / 1\right)$ still converges by Theorem 3.5A.

B. Let $K\left(a_{n} / 1\right)$ be from $\left\langle\tilde{E}_{1, \delta}, E_{2}\right\rangle$ and let $0 \notin \partial^{\dagger} V_{1}$. If $\tilde{E}_{2, \delta}=E_{2}$, then the situation is covered by part $\mathrm{B}$, so let $R_{0}=\left|1+C_{0}\right|$ and $h \geq 0$. That is, $-1 \in \partial V_{0}$ and $0 \notin V_{1}^{\circ}$, and so, $0 \notin V_{1}$ under our conditions. Now, $a_{2 n-1} \in \tilde{E}_{1, \delta} \in \mathcal{E}\left(V_{1}, V_{0}\right)$ by Lemma 4.4 , so $\left\langle\tilde{E}_{1, \delta}, E_{2}\right\rangle \in \mathcal{E}\left(V_{0}\right)$. The result follows therefore from Theorem $3.5 \mathrm{~A}$ since $0 \notin V_{1}$ implies that $-1 \notin \partial^{*} V_{0}$, and thus $-1 \notin Z_{0}$.

C. Let $K\left(a_{n} / 1\right)$ be from $\left\langle\tilde{E}_{1, \delta}, E_{2}\right\rangle$. By Lemma $4.4,\left\langle\tilde{E}_{1, \delta}, E_{2}\right\rangle \in \mathcal{E}\left(V_{0}\right)$. Hence $Z_{0} \subseteq \partial^{*} V_{0}$ by Theorem 3.5. The convergence follows therefore from Theorem 3.5A.

D. That $c_{2 n} \rightarrow c$ follows from Theorem 1.4D. We know that $Z_{k} \subseteq\left(-1-V_{k+1}\right) \backslash V_{k}^{\circ}$ by Theorem $1.4 \mathrm{~A}$. Therefore $0 \notin Z_{1}$ if $0 \in V_{1}^{\circ}$ or $-1 \notin V_{0}$, which in our situation holds if $-1 \notin \partial V_{0}$, and $c_{n} \rightarrow c$ by Theorem 1.4E.

The conditions on $\left\{a_{n}\right\}$ imply that $\operatorname{dist}\left(a_{2 n-1}, Z_{0}\right) \geq \varepsilon$ from some $n$ on (Theorem $3.5)$, and thus $c_{2 n-1} \rightarrow c$ by Theorem $1.4 \mathrm{C}$.

Lemma 4.5 (The disk - complement of disk case). Let $V_{0}:=B\left(C_{0}, R_{0}\right)$ and $V_{1}:=\overline{B\left(C_{1}, R_{1}\right)^{c}}$ where $C_{0}, C_{1} \in \mathbb{C}$ and $R_{0}, R_{1}>0$ satisfy $(2.9)$. Let $E_{k}$ and $\widehat{E}_{k, \delta}$ be given as in Theorem 2.5. Then $\left\langle E_{1}, E_{2}\right\rangle$ are the element sets corresponding to $\left\langle V_{0}, V_{1}\right\rangle$, and $\left\langle\widehat{E}_{1, \delta}, E_{2}\right\rangle \in \mathcal{E}\left(V_{0}\right)$ and $\left\langle\widehat{E}_{2, \delta}, E_{1}\right\rangle \in \mathcal{E}\left(V_{1}\right)$.

Proof. For $a \neq 0$ the set $a /\left(1+V_{1}\right)$ is a circular disk $B\left(\widehat{C}_{0}, \widehat{R}_{0}\right)$ where

$$
\widehat{C}_{0}=\frac{a\left(1+\overline{C_{1}}\right)}{\left|1+C_{1}\right|^{2}-R_{1}^{2}}, \quad \widehat{R}_{0}=\frac{|a| R_{1}}{R_{1}^{2}-\left|1+C_{1}\right|^{2}} .
$$

It is $\subseteq V_{0}$ if and only if $\left|\widehat{C}_{0}-C_{0}\right|+\widehat{R}_{0} \leq R_{0}$, i.e., if and only if $a \in E_{1}$. It is equal to $V_{0}$ if and only if $\widehat{C}_{0}=C_{0}$ and $\widehat{R}_{0}=R_{0}$, i.e., if and only if either

$$
\begin{aligned}
C_{1} \neq-1, \quad a=\tilde{a}_{1} & \text { and }\left|C_{0}\right| R_{1}=R_{0}\left|1+C_{1}\right| \\
& \text { or } C_{1}=-1, C_{0}=0 \text { and }|a|=R_{0} R_{1} .
\end{aligned}
$$

Since $\widehat{E}_{1, \delta}$ is a closed, bounded set in $\mathbb{C}$ with $a /\left(1+V_{1}\right) \neq V_{0}$ for all $a \in \widehat{E}_{1, \delta}$, we have $\widehat{E}_{1, \delta} \in \mathcal{E}\left(V_{1}, V_{0}\right)$. Since $s_{1} \circ s_{2}\left(V_{0}\right) \subseteq s_{1}\left(V_{1}\right)$ this proves that $\left\langle\widehat{E}_{1, \delta}, E_{2}\right\rangle \in \mathcal{E}\left(V_{0}\right)$.

Let $\left|1+C_{0}\right|<R_{0}$. Then $a /\left(1+V_{0}\right)$ is the exterior of a disk. Indeed, $a /\left(1+V_{0}^{\circ}\right)=$ $B\left(\widehat{C}_{1}, \widehat{R}_{1}\right)^{c}$ where

$$
\widehat{C}_{1}=\frac{a\left(1+\overline{C_{0}}\right)}{\left|1+C_{0}\right|^{2}-R_{0}^{2}}, \quad \widehat{R}_{1}=\frac{|a| R_{0}}{R_{0}^{2}-\left|1+C_{0}\right|^{2}} .
$$

It is $\subseteq V_{1}$ if and only if $\left|\widehat{C}_{1}-C_{1}\right|+R_{1} \leq \widehat{R}_{1}$, i.e., if and only if $a \in E_{2}$. It is equal to $V_{1}^{\circ}$ if and only if $\widehat{C}_{1}=C_{1}$ and $\widehat{R}_{1}=R_{1}$, i.e., if and only if either

$$
\begin{aligned}
-1 \in V_{0}^{\circ}, C_{0} \neq-1, & a=\tilde{a}_{2} \text { and }\left|C_{1}\right| R_{0}=R_{1}\left|1+C_{0}\right| \\
& \text { or } C_{0}=-1, C_{1}=0 \text { and }|a|=R_{0} R_{1} .
\end{aligned}
$$


These cases are excluded for $a \in \widehat{E}_{2, \delta}$. From (2.10) we see that $0 \notin \overline{E_{2}}$ when $\left|1+C_{0}\right|<R_{0}$. We need to check whether $a_{2 n_{k}} /\left(1+V_{0}\right) \rightarrow V_{1}$ is possible for $a_{2 n_{k}} \in E_{2}$ if $a_{2 n_{k}} \rightarrow \infty$. But this is no problem since $V_{0}$ is bounded, and thus $\lim _{a \rightarrow \infty} a /\left(1+V_{0}\right)=\{\infty\}$. Therefore $\tilde{E}_{2, \delta} \in \mathcal{E}\left(V_{0}, V_{1}\right)$, and thus $\left\langle\widehat{E}_{2, \delta}, E_{1}\right\rangle \in \mathcal{E}\left(V_{1}\right)$.

Next, let $\left|1+C_{0}\right|=R_{0}$. Then for $a \neq 0, a /\left(1+V_{0}\right)$ is the half plane given by (4.6). Hence $\left\langle E_{2}, E_{1}\right\rangle \in \mathcal{E}\left(V_{1}\right)$ and $a /\left(1+V_{0}\right) \subseteq V_{1}$ if and only if

$$
\operatorname{Re}\left(C_{1} \frac{1+C_{0}}{\left|1+C_{0}\right|} e^{-i \theta}\right)+R_{1} \leq \frac{|a|}{2 R_{0}} \quad \text { where } \theta:=\arg a,
$$

which gives the expression for $E_{2}$ in this case. $\left(0 \notin E_{2}\right.$ since $-1 \in V_{0}$.)

Finally, let $\left|1+C_{0}\right|>R_{0}$. Then $a /\left(1+V_{0}\right)=B\left(\widehat{C}_{1},-\widehat{R}_{1}\right)$ for $a \neq 0$, where $\widehat{C}_{1}$ and $\widehat{R}_{1}$ are given by (4.10). Therefore $a /\left(1+V_{0}\right) \subseteq V_{1}$ if and only if $\left|\widehat{C}_{1}-C_{1}\right| \geq R_{1}+\left|\widehat{R}_{1}\right|$, i.e., if and only if $a \in E_{2}$. Moreover, $\left\langle E_{2}, E_{1}\right\rangle \in \mathcal{E}\left(V_{1}\right)$.

Proof of Theorem 2.5. A. The expressions for $E_{1}$ and $E_{2}$ follow from Lemma 4.5. We need to check that $E_{k}^{\circ} \neq \emptyset$ for $k=1,2$. This clearly holds for $E_{1}$ since $\left|C_{0}\right|<R_{0}$ and $\left|1+C_{1}\right|<R_{1}$, and thus $0 \in E_{1}^{\circ}$. It is also clear that $E_{2}^{\circ} \neq \emptyset$ if $C_{0}=-1$ or if $R_{0}=\left|1+C_{0}\right|$. Let $R_{0}<\left|1+C_{0}\right|$ and $C_{1} \neq 0$. Then $\tilde{a}_{2} \neq 0$ and $-t \tilde{a}_{2} \in E_{2}^{\circ}$ for all $t>0$ sufficiently large. If $R_{0}<\left|1+C_{0}\right|$ and $C_{1}=0$, then $\tilde{a}_{2}=0$ and $E_{2}=\left\{a ;|a| \geq R_{1}\left(\left|1+C_{0}\right|+R_{0}\right)\right\}$, so again $E_{2}^{\circ} \neq \emptyset$. If $R_{0}>\left|1+C_{0}\right|>0$ and $C_{1} \neq 0$, then $t \tilde{a}_{2} \in E_{2}^{\circ}$ for all $t>0$ sufficiently large, and thus $E_{2}^{\circ} \neq \emptyset$. Finally, if $R_{0}>\left|1+C_{0}\right|$ and $C_{1}=0$, then $\tilde{a}_{2}=0$ and all $a$ with $|a| \geq R_{0}^{2}-\left|1+C_{0}\right|^{2}$ are $\in E_{2}$.

Let $K\left(a_{n} / 1\right)$ be a continued fraction from $\left\langle E_{1}, E_{2}\right\rangle$. If $\operatorname{rad}\left(S_{2 n}\left(V_{0}\right)\right) \rightarrow 0$ or $\operatorname{rad}\left(S_{2 n}\left(W_{0}\right)\right) \rightarrow 0$ or $\operatorname{diam}\left(S_{2 n}\left(Y_{0}\right)\right) \rightarrow 0$, then $K\left(a_{n} / 1\right)$ clearly converges generally. Assume in the proof of parts B and C below that $\operatorname{diam}\left(S_{2 n}\left(Y_{0}\right)\right) \rightarrow \tilde{d}>0$, and thus $\operatorname{rad}\left(S_{2 n}\left(V_{0}\right)\right) \rightarrow R>0$ and $\operatorname{rad}\left(S_{2 n}\left(W_{0}\right)\right) \rightarrow R^{*}>0$.

B. We first observe that $W_{0}=B\left(-1-C_{1}, R_{1}\right)$ and $W_{1}=\overline{B\left(-1-C_{0}, R_{0}\right)^{c}}$ in this case. By Lemma 4.1 the element sets $E_{1}$ and $E_{2}$ do not change if we replace $\left\langle V_{0}, V_{1}\right\rangle$ by $\left\langle W_{0}, W_{1}\right\rangle$ (although their representation (2.10) changes), and neither do the conditions in (2.11). Indeed, $\widehat{E}_{1, \delta}$ and $\widehat{E}_{2, \delta}$ do not change either, since

$$
\tilde{a}_{k}=C_{k-1}\left(1+C_{k}\right)\left(1-R_{k}^{2} /\left|1+C_{k}\right|^{2}\right)=\left(-1-C_{k}\right)\left(-C_{k-1}\right)\left(1-R_{k-1}^{2} /\left|C_{k-1}\right|^{2}\right)
$$

when $\left|C_{k-1}\right| R_{k}=R_{k-1}\left|1+C_{k}\right|>0$. Therefore $\widehat{E}_{1, \delta} \in \mathcal{E}\left(W_{1}, W_{0}\right) \cap \mathcal{E}\left(V_{1}, V_{0}\right)$ by Lemma 4.5.

There is one condition that is changed, though, and that is the condition $-1 \notin$ $V_{0}^{\circ}$, which is equivalent to $0 \in W_{1}$. This means that if $-1 \notin V_{0}^{\circ}$, then $E_{2} \in$ $\mathcal{E}\left(V_{0}, V_{1}\right)$, whereas, by (4.11), $E_{2} \notin \mathcal{E}\left(W_{0}, W_{1}\right)$ if also $-1 \in W_{0}^{\circ}$ and $\left|C_{1}\right| R_{0}=$ $R_{1}\left|1+C_{0}\right| \geq 0$. However, this case cannot occur since

$$
-1 \notin V_{0}^{\circ} \Leftrightarrow\left|1+C_{0}\right| \geq R_{0} \quad \text { and } \quad-1 \in W_{0}^{\circ} \Leftrightarrow\left|C_{1}\right|<R_{1},
$$

which give $\left|C_{1}\right| R_{0}<R_{1}\left|1+C_{0}\right|$. Therefore, also now $\widehat{E}_{2, \delta} \in \mathcal{E}\left(W_{0}, W_{1}\right) \cap \mathcal{E}\left(V_{0}, V_{1}\right)$ by Lemma 4.5. This means that $Z_{k} \in \partial^{\dagger} V_{k}$ by Theorem 3.5B. Since $\partial^{\dagger} V_{0}=-1-\partial^{\dagger} V_{1}$, the convergence follows from Theorem $3.5 \mathrm{~A}$, both if $K\left(a_{n} / 1\right)$ is from $\left\langle\widehat{E}_{1, \delta}, E_{2}\right\rangle$ or from $\left\langle E_{1}, \widehat{E}_{2, \delta}\right\rangle$.

C. By the proof of part B, $Z_{0} \subseteq \partial^{\dagger} V_{0}$ when $K\left(a_{n} / 1\right)$ is from $\left\langle\widehat{E}_{1, \delta}, E_{2}\right\rangle$, and $Z_{1} \subseteq \partial^{\dagger} V_{1}$ when $K\left(a_{n} / 1\right)$ is from $\left\langle E_{1}, \widehat{E}_{2, \delta}\right\rangle$. The result follows therefore from Theorem 3.5A. 
D: Let $K\left(a_{n} / 1\right)$ from $\left\langle E_{1}, E_{2}\right\rangle$ converge generally to $c$. Then $c_{2 n} \rightarrow c$ and $\tilde{Z}_{k}=$ $Z_{k}$ for $k=0,1$ by Theorem 1.4D. Therefore $Z_{0} \subseteq \overline{V_{0}^{c}} \cap\left(-1-V_{1}\right)$ (Theorem 1.4A). It follows therefore from Theorem 1.4E and $\mathrm{C}$ with $k=0$ that also $c_{2 n-1} \rightarrow c$.

Proof of Theorem 2.7. Let $k \in\{1,2\}$ be fixed. First let $-1 \notin \partial V_{k}$. Then $g_{k}<1$, $\left|\alpha_{k}\right|<\pi / 2$ and

$$
a /\left(1+V_{k}\right)=B\left(\tilde{C}_{k}, \tilde{R}_{k}\right), \quad \tilde{C}_{k}:=\frac{a e^{-i \alpha_{k}}}{2\left(1-g_{k}\right) \cos \alpha_{k}}, \quad \tilde{R}_{k}:=\frac{|a|}{2\left(1-g_{k}\right) \cos \alpha_{k}}
$$

for $a \neq 0$. This set is contained in $V_{k-1}$ if and only if $\operatorname{Re}\left(\tilde{C}_{k} e^{-i \alpha_{k-1}}\right)-\tilde{R}_{k} \geq$ $-g_{k-1} \cos \alpha_{k-1}$, which proves the expression for $E_{k}$ in this case. Next let $-1 \in \partial V_{k}$. Then $1 /\left(1+V_{k}\right)=H\left(0,-\alpha_{k}\right)$. Hence $a /\left(1+V_{k}\right) \subseteq V_{k-1}$ for $a \neq 0$ if and only if $\arg (a)=\alpha_{k-1}+\alpha_{k}$. Since either $g_{k}=1$ or $\left|\alpha_{k}\right|=\pi / 2$ when $-1 \in \partial V_{k}$, the expression (2.15) for $E_{k}$ is still valid. Therefore $\left\langle E_{1}, E_{2}\right\rangle$ given by (2.15) are the element sets corresponding to $\left\langle V_{0}, V_{1}\right\rangle$.

If $0,-1 \notin V_{k}$ for both $k=0$ and $k=1$, then the convergence follows from the twin version of the multiple parabola theorem proved in [5]. (See Remark 2.8.3.) Otherwise, by (2.14), there exist $\tilde{g}_{0}, \tilde{g}_{1}, \tilde{\alpha}_{0}$ and $\tilde{\alpha}_{1}$ such that

$$
\begin{aligned}
& \left|\tilde{\alpha}_{0}\right|<\frac{\pi}{2}, \quad\left|\tilde{\alpha}_{1}\right|<\frac{\pi}{2} \quad \text { and } \tilde{\alpha}_{0}+\tilde{\alpha}_{1}=\alpha_{0}+\alpha_{1}, \\
& 0<\tilde{g}_{0}<1, \quad 0<\tilde{g}_{1}<1 \quad \text { and } \tilde{g}_{k}\left(1-\tilde{g}_{k-1}\right) \geq g_{k}\left(1-g_{k-1}\right) \text { for } k=1,2 .
\end{aligned}
$$

Let $\tilde{E}_{1}$ and $\tilde{E}_{2}$ be given by (2.15) with $g_{0}, g_{1}, \alpha_{0}$ and $\alpha_{1}$ replaced by $\tilde{g}_{0}, \tilde{g}_{1}, \tilde{\alpha}_{0}$ and $\tilde{\alpha}_{1}$. Then $E_{1} \subseteq \tilde{E}_{1}$ and $E_{2} \subseteq \tilde{E}_{2}$, and the convergence follows again from the twin version of the multiple parabola theorem.

Proof of Theorem 1.3. Since $\left|C_{k}\right| R_{k+1} \neq R_{k}\left|1+C_{k+1}\right|$ for $k=0$ or $k=1$, we have $\widehat{E}_{k+1, \delta}=E_{k+1}$ in $(2.11)$ for this $k$, and $K\left(a_{n} / 1\right)$ converges generally by Theorem $2.5 \mathrm{~B}$.

Proof of Theorem 1.1. A. Since $E_{2}^{\circ}=\emptyset$ if and only if $E_{2}=\left\{\tilde{a}_{2}\right\}$ in this case, which happens if and only if $\left|C_{1}\right| R_{0}=\left|1+C_{0}\right| R_{1}$, it follows from Theorem 2.1 that $K\left(a_{n} / 1\right)$ converges.

B. By $(2.7)$ we always have $-1 \notin V_{0}$ when $E_{2}^{\circ} \neq \emptyset$. Hence $\tilde{E}_{2, \delta}=E_{2}$, and $K\left(a_{n} / 1\right)$ converges generally by Theorem $2.3 \mathrm{~B}$. Theorem $1.4 \mathrm{D}$ shows therefore that its even part converges, and Theorem $1.4 \mathrm{E}$ shows that its odd part converges.

C. It follows from Theorem 1.3 that $K\left(a_{n} / 1\right)$ converges generally in this case. Therefore its even part converges by Theorem 1.4D. The convergence of $K\left(a_{n} / 1\right)$ follows from Theorem 1.4E.

D. (2.14) holds under our conditions, and the result follows from Theorem 2.7.

\section{REFERENCES}

1. Jacobsen, Lisa, Some periodic sequences of circular convergence regions, Lecture Notes in Math. No 932, Springer-Verlag (1982), 87-98. MR690456 (84c:40003)

2. Jacobsen, Lisa, General convergence of continued fractions, Trans. Amer. Math. Soc. 294(2) (1986), 477-485. MR825716 (87j:40004)

3. Jacobsen, Lisa and Thron, W.J., Oval Convergence Regions and Circular Limit Regions for Continued Fractions $K\left(a_{n} / 1\right)$, Lecture Notes in Math., Springer-Verlag 1199 (1986), 90-126. MR $870246(88 \mathrm{c}: 40004)$

4. Jacobsen, Lisa and Thron, W.J., Limiting structures for sequences of linear fractional transformations, Proc. Amer. Math. Soc. 99 (1987), 141-146. MR866444 (88d:40010) 
5. Jones, William B. and Thron, W.J., Convergence of continued fractions, Can. J. Math. 20 (1968), 1037-1055. MR0230888 (37:6446)

6. Jones, William B. and Thron, W.J., Twin-convergence regions for continued fractions $K\left(a_{n} / 1\right)$, Trans. Amer. Math. Soc. 150 (1970), 93-119. MR.0264043 (41:8640)

7. Jones, William B. and Thron, W.J., Continued Fractions. Analytic Theory and Applications. Encyclopedia of Mathematics and Its Applications 11, Addison-Wesley Publishing Company, 1980. MR 0595864 (82c:30001)

8. Lane, R.E., The Convergence and Values of Periodic Continued Fractions, Bull. Amer. Math. Soc. 51 (1945), 246-250. MR0011748 (6:211b)

9. Lorentzen, Lisa, Convergence criteria for continued fractions $K\left(a_{n} / 1\right)$ based on value sets, Contemporary Mathematics 236 (1999), 205-255. MR1665372 (2000i:30006)

10. Lorentzen, Lisa, Möbius transformations mapping the unit disk into itself, The Ramanujan J. Math. 13 (2007), 253-263. MR.2281165

11. Lorentzen, Lisa and Ruscheweyh, St., Simple convergence sets for continued fractions $K\left(a_{n} / 1\right)$, Math. Anal. and Appl. 179(2) (1993), 349-370. MR1249825 (95b:40001)

12. Lorentzen, Lisa and Waadeland, Haakon, Continued Fractions with Applications, Studies in Computational Mathematics 3, Elsevier Science Publishers B.V., 1992. MR 1172520 (93g:30007)

Lisa Lorentzen has changed her name from Lisa Jacobsen.

Department of Mathematical Sciences, Norwegian University of Science and TechNOLOGY, N-7491 TrondHEIM, NORWAY 